\title{
A NEW APPROACH TO TRANSPORT EQUATIONS ASSOCIATED TO A REGULAR FIELD: TRACE RESULTS AND WELL-POSEDNESS.
}

\author{
L. ARLOTTI, J. BANASIAK \& B. LODS
}

\begin{abstract}
We generalize known results on transport equations associated to a Lipschitz field $\mathscr{F}$ on some subspace of $\mathbb{R}^{N}$ endowed with some general space measure $\mu$. We provide a new definition of both the transport operator and the trace measures over the incoming and outgoing parts of $\partial \boldsymbol{\Omega}$ generalizing known results from $[9,16]$. We also prove the well-posedness of some suitable boundary-value transport problems and describe in full generality the generator of the transport semigroup with no-incoming boundary conditions.
\end{abstract}

\section{INTRODUCTION}

In this paper we present new methodological tools to investigate the well-posedness of the general transport equation

$$
\partial_{t} f(\mathbf{x}, t)+\mathscr{F}(\mathbf{x}) \cdot \nabla_{\mathbf{x}} f(\mathbf{x}, t)=0 \quad(\mathbf{x} \in \boldsymbol{\Omega}, t>0),
$$

supplemented by boundary condition

$$
f_{\mid \Gamma_{-}}(\mathbf{y}, t)=\psi_{-}(\mathbf{y}, t), \quad\left(\mathbf{y} \in \Gamma_{-}, t>0\right),
$$

and the initial condition

$$
f(\mathbf{x}, 0)=f_{0}(\mathbf{x}), \quad(\mathbf{x} \in \mathbf{\Omega}) .
$$

Here $\Omega$ is a sufficiently smooth open subset of $\mathbb{R}^{N}, \Gamma_{ \pm}$are suitable boundaries of the phase space and $\psi_{-}$is a given function of the trace space $L^{1}\left(\Gamma_{-}, \mathrm{d} \mu_{-}\right)$corresponding to the boundary $\Gamma_{-}$(see Section 2 for details).

The present paper is part of a series of papers on transport equations with general vector fields $[5,6]$ and introduce all the methodological tools that allow us not only to solve the initial-boundary problem (1.1) but also to treat in [6] the case of abstract boundary conditions relying the incoming and outgoing fluxes, generalizing the results of [9].

The main novelty of our approach is that we assume $\mathbb{R}^{N}$ to be endowed with a general positive Radon measure $\mu$. Here by a Radon measure we understand a Borel measure (or its completions, see $[15$, p. 332]) which is finite on compact sets. As we shall see it further on, taking into account such general Radon measure $\mu$ leads to a large amount of technical difficulties, in particular in the definition of trace spaces and in the derivation of Green's formula. Moreover, for such a measure $\mu$, it is far from being trivial to identify the vector field $\mathscr{F} \cdot \nabla_{x}$ to the time derivative along the characteristic curves (as done in [9, Formulae (5.4) \& (5.5), p.392]): the main difficulty stemming from the impossibility of applying classical convolution arguments (and the so-called Friedrich's lemma). We overcome this difficulty by introducing new mollification techniques along the characteristic curves. Let us explain in more details our general assumptions:

Keywords: Transport equation, Boundary conditions, $C_{0}$-semigroups, Characteristic curves.

AMS subject classifications (2000): 47D06, 47D05, 47N55, 35F05, 82C40. 
1.1. General assumption and motivations. The transport coefficient $\mathscr{F}$ is a time independent vector field $\mathscr{F}: \mathbb{R}^{N} \longrightarrow \mathbb{R}^{N}$ which is (globally) Lipschitz-continuous with Lipschitz constant $\kappa>0$, i.e.

$$
\left|\mathscr{F}\left(\mathbf{x}_{1}\right)-\mathscr{F}\left(\mathbf{x}_{2}\right)\right| \leqslant \kappa\left|\mathbf{x}_{1}-\mathbf{x}_{2}\right| \quad \text { for any } \mathbf{x}_{1}, \mathbf{x}_{2} \in \mathbb{R}^{N} .
$$

Clearly, one can associate a flow $\left(T_{t}\right)_{t \in \mathbb{R}}$ to this field $\mathscr{F}$ (with the notations of Section 2.1, $T_{t}=$ $\Theta(\cdot, t, 0)$ ) and we make the following fundamental assumption (known as Liouville's Theorem whenever $\mu$ is the Lebesgue measure) on $\mathscr{F}$ :

Assumption 1. The measure $\mu$ is invariant under the flow $\left(T_{t}\right)_{t \in \mathbb{R}}$, i.e. $\mu\left(T_{t} A\right)=\mu(A)$ for any measurable subset $A \subset \mathbb{R}^{N}$ and any $t \in \mathbb{R}$.

Remark 1.1. Notice that, whenever $\mu$ is the Lebesgue measure over $\mathbb{R}^{N}$, it is well-known that Assumption 1 is equivalent to $\operatorname{div}(\mathscr{F}(\mathbf{x}))=0$ for any $\mathbf{x} \in \mathbb{R}^{N}$. More generally, by virtue of $[2$, Remark 3 \& Proposition 4], Assumption 1 holds for a general Borel measure $\mu$ provided the field $\mathscr{F}$ is locally integrable with respect to $\mu$ and divergence-free with respect to $\mu$ in the sense that

$$
\int_{\mathbb{R}^{N}} \mathscr{F}\left(T_{t}(\mathbf{x})\right) \cdot \nabla_{\mathbf{x}} f\left(T_{t}(\mathbf{x})\right) \mathrm{d} \mu(\mathbf{x})=0, \quad \forall t \in \mathbb{R}
$$

for any infinitely differentiable function $f$ with compact support.

A typical example of such a transport equation is the so-called Vlasov equation for which:

i) The phase space $\Omega$ is given by the cylindrical domain $\Omega=\mathcal{D} \times \mathbb{R}^{3} \subset \mathbb{R}^{6}$ where $\mathcal{D}$ is a sufficiently smooth open subset of $\mathbb{R}^{3}$, referred to as the position space, while the so-called velocity space is here given by $\mathbb{R}^{3}$. The measure $\mu$ is given by $\mathrm{d} \mu(\mathbf{x})=\mathrm{d} x \mathrm{~d} \beta(v)$ where $\beta$ is a suitable Radon measure on $\mathbb{R}^{3}$, e.g. Lebesgue measure over $\mathbb{R}^{3}$ for continuous models or combination of Lebesgue measures over suitable spheres for the multigroup model.

ii) For any $\mathbf{x}=(x, v) \in \mathcal{D} \times \mathbb{R}^{3}$,

$$
\mathscr{F}(\mathbf{x})=(v, \mathbf{F}(x, v)) \in \mathbb{R}^{6}
$$

where $\mathbf{F}=\left(\mathbf{F}_{1}, \mathbf{F}_{2}, \mathbf{F}_{3}\right)$ is a time independent force field over $\mathcal{D} \times \mathbb{R}^{3}$ satisfying Assumption 1 and (1.2). The free transport case, investigated in [16, 4], corresponds to $\mathbf{F}=0$.

The existence of solution to the transport equation (1.1a) is a classical matter when considering the whole space $\Omega=\mathbb{R}^{N}$. In particular, the concept of renormalized solutions allows to consider irregular transport coefficient $\mathscr{F}(\cdot)$ (see [10] and the recent contributions [2, 13]) which is of particular relevance in fluid mechanics.

On the other hand, there are few results addressing the initial-boundary value problem (1.1), possibly due to difficulties created by the boundary conditions (1.1b). We mention here the seminal works by C. Bardos [8], and by R. Beals and V. Protopopescu [9] (see also [11, 14]). Let us however mention that the results of $[9,11]$ introduce restrictive assumptions on the characteristics of the equation. For instance, fields with 'too many' periodic trajectories create serious difficulties. They are however covered in a natural way by the theory presented here, see Examples $2.5 \& 2.6$.

1.2. Presentation of the results. In this paper, we revisit and generalize the afore-mentioned results to the general case $\mathbf{F} \neq 0$ and for a general Radon measure $\mu$. The latter, in particular, leads to numerous technical problems such as e.g. determination of suitable measures $\mu_{ \pm}$over the 'incoming' and 'outgoing' parts $\Gamma_{ \pm}$of $\partial \boldsymbol{\Omega}$. We provide here a general construction of these 'trace measures' generalizing, and making more precise, the results of $[9,11]$. This construction allows us to establish Proposition 2.12 which allows to compute integrals over $\Omega$ via integration along 
the integral curves of $\mathscr{F}(\cdot)$ coming from the boundary $\partial \boldsymbol{\Omega}$, and which is free from some restrictive assumptions of op. cit. In particular, we present a new proof of the Green formula clarifying and removing gaps of the proofs in $[9,11]$. Of course, the boundary condition $(1.1 b)$ we treat here is less general than the abstract ones investigated in $[9,11]$ but, as we already mentioned it, the tools we introduce here will allow us to generalize, in a subsequent paper [6], the results of the op. cited by dealing with abstract boundary conditions.

Another major difficulty, when dealing with a general Radon measure $\mu$, is to provide a precise definition of the transport operator $\mathcal{T}_{\max }$ associated to (1.1). It appears quite natural to define the transport operator $\mathcal{T}_{\max }$ (with its maximal domain on $L^{1}(\Omega, \mathrm{d} \mu)$ ) as a weak directional derivative along the characteristic curves in the $L^{1}$-sense. However, it is not clear a priori that any function $f$ for which the weak directional derivative exists in $L^{1}(\Omega, \mathrm{d} \mu)$ (with appropriate and minimal class of test-functions) admits a trace over $\Gamma_{ \pm}$. With the aim of proving such a trace result, we provide here a new characterization of the transport operator related to a mild representation of the solution to (1.1). Namely, we prove (Theorem 3.6) that the domain $\mathscr{D}\left(\mathcal{T}_{\max }\right)$ (as defined in Section 3), is precisely the set of functions $f \in L^{1}(\Omega, \mathrm{d} \mu)$ that admits a representative which is absolutely continuous along almost any characteristic curve.

Note that in the classical case when $\mu$ is the Lebesgue measure, such a representation is known to be true [10, Appendix]. Actually, in this case, one defines the domain $\mathscr{D}\left(\mathcal{T}_{\max }\right)$ as the set of all $f \in L^{1}(\Omega, \mathrm{d} \mu)$ for which the directional derivative $-\mathscr{F} \cdot \nabla f$ exists in the distributional sense and belongs to $L^{1}(\Omega, \mathrm{d} \mu)$. Then, by convolution arguments, it is well-known that the set $\mathscr{C}_{0}^{1}(\boldsymbol{\Omega}) \cap \mathscr{D}\left(\mathcal{T}_{\max }\right)$ is dense in $\mathscr{D}\left(\mathcal{T}_{\max }\right)$ for the graph norm $\|f\|=\|f\|+\|\mathscr{F} \cdot \nabla f\|$.

The question is much more delicate for a general Radon measure $\mu$. Indeed, in such a case, the convolution argument used in the case of the Lebesgue measure does not apply anymore. Our strategy to prove the characterization of $\mathcal{T}_{\max }$ is also based on a convolution argument but it uses mollification technique along the characteristic curves as developed in Section 3. Such a result shall allow us to obtain a rigorous derivation of Green's formula, clarifying some results of [9].

1.3. Plan of the paper. The organization of the paper is as follows. In Section 2 we introduce main tools used throughout the paper and present the aforementioned new results concerning integration over the characteristic curves of $\mathscr{F}$ as well as a new construction of the boundary measures over the 'incoming' and 'outgoing' parts $\Gamma_{ \pm}$of $\partial \boldsymbol{\Omega}$ which generalizes and clarifies that of $[9,11]$. In Section 3 we provide a construction of the maximal transport operator $\mathcal{T}_{\max }$. It is defined in a weak sense, through its action on suitably defined test functions. The fundamental result of this section shows that any function in the domain $\mathscr{D}\left(\mathcal{T}_{\max }\right)$ admits a representation which is absolutely continuous along almost any characteristic which, in turn, allows for existence of its traces on the outgoing and incoming parts of the boundary. In Section 4 we apply the results of Section 3 to prove well-posedness of the time-dependent transport problem with no reentry boundary conditions associated with $\mathcal{T}_{\max }$. Moreover, we consider the corresponding stationary problem and, as a by-product, we recover a new proof of the Green formula.

\section{INTEGRATION ALONG THE CHARACTERISTICS}

2.1. Characteristic curves. A crucial role in our study is played by the characteristic curves associated to the field $\mathscr{F}$. Precisely, for any $\mathbf{x} \in \mathbb{R}^{N}$ and $t \in \mathbb{R}$, consider the initial-value problem

$$
\left\{\begin{array}{l}
\frac{\mathrm{d}}{\mathrm{d} s} \mathbf{X}(s)=\mathscr{F}(\mathbf{X}(s)), \quad(s \in \mathbb{R}) \\
\mathbf{X}(t)=\mathbf{x}
\end{array}\right.
$$


Since $\mathscr{F}$ is Lipschitz continuous on $\mathbb{R}^{N}$, Eq. (2.1) has a unique global in time solution and this allows to define the flow-mapping $\Theta: \mathbb{R}^{N} \times \mathbb{R} \times \mathbb{R} \rightarrow \mathbb{R}^{N}$, such that, for $(\mathbf{x}, t) \in \mathbb{R}^{N} \times \mathbb{R}$, the mapping:

$$
\mathbf{X}(\cdot): s \in \mathbb{R} \longmapsto \mathbf{\Theta}(\mathbf{x}, t, s)
$$

is the only solution of Eq. (2.1). Being concerned with solutions to the transport equation (1.1) in the region $\Omega$, we have to introduce the definition of stay times of the characteristic curves in $\Omega$ :

Definition 2.1. For any $\mathrm{x} \in \boldsymbol{\Omega}$, define $\tau_{ \pm}(\mathrm{x})=\inf \{s>0 ; \boldsymbol{\Theta}(\mathrm{x}, 0, \pm s) \notin \boldsymbol{\Omega}\}$, with the convention that inf $\varnothing=\infty$, and $\operatorname{set} \tau(\mathbf{x})=\tau_{+}(\mathbf{x})+\tau_{-}(\mathbf{x})$.

In other words, given $\mathbf{x} \in \Omega, I_{\mathbf{x}}=\left(-\tau_{-}(\mathbf{x}), \tau_{+}(\mathbf{x})\right)$ is the maximal interval for which $\boldsymbol{\Theta}(\mathbf{x}, 0, s)$ lies in $\boldsymbol{\Omega}$ for any $s \in I_{\mathbf{x}}$ and $\tau(\mathbf{x})$ is the length of the interval $I_{\mathbf{x}}$. Notice that $0 \leqslant \tau_{ \pm}(\mathbf{x}) \leqslant \infty$. Thus, the function $\Theta$ restricted to the set

$$
\boldsymbol{\Lambda}:=\left\{(\mathbf{x}, t, s) ; \mathbf{x} \in \mathbf{\Omega}, t \in \mathbb{R}, s \in\left(t-\tau_{-}(\mathbf{x}), t+\tau_{+}(\mathbf{x})\right)\right\}
$$

is such that $\boldsymbol{\Theta}(\boldsymbol{\Lambda})=\boldsymbol{\Omega}$. Note that here we do not assume that the length of the interval $I_{\mathrm{x}}=$ $\left(-\tau_{-}(\mathbf{x}), \tau_{+}(\mathbf{x})\right)$ is finite. In particular, $I_{\mathbf{x}}=\mathbb{R}$ for any stationary point $\mathbf{x}$ of $\mathscr{F}$, i.e. $\mathscr{F}(\mathbf{x})=0$. If $\tau(\mathbf{x})$ is finite, then the function $\mathbf{X}: s \in I_{\mathbf{x}} \longmapsto \boldsymbol{\Theta}(\mathbf{x}, 0, s)$ is bounded since $\mathscr{F}$ is Lipschitz continuous. Moreover, still by virtue of the Lipschitz continuity of $\mathscr{F}$, the only case when $\tau_{ \pm}(\mathbf{x})$ is finite is when $\boldsymbol{\Theta}(\mathbf{x}, 0, \pm s)$ reaches the boundary $\partial \boldsymbol{\Omega}$ so that $\boldsymbol{\Theta}\left(\mathbf{x}, 0, \pm \tau_{ \pm}(\mathbf{x})\right) \in \partial \boldsymbol{\Omega}$. We note that, since $\mathscr{F}$ is Lipschitz around each point of $\partial \boldsymbol{\Omega}$, the points of the set $\{\mathbf{y} \in \partial \boldsymbol{\Omega} ; \mathscr{F}(\mathbf{y})=0\}$ (introduced in $[9,11]$ ) are equilibrium points of the $\mathscr{F}$ and cannot be reached in finite time.

Remark 2.2. We emphasize that periodic trajectories which do not meet the boundaries have $\tau_{ \pm}=\infty$ and thus are treated as infinite though geometrically they are bounded.

Finally we mention that it is not difficult to prove that the mappings $\tau_{ \pm}: \Omega \rightarrow \mathbb{R}^{+}$are lower semicontinuous and therefore measurable, see e.g., [7, p. 301]

The flow $\boldsymbol{\Theta}(\mathbf{x}, t, s)$ defines, at each instant $t$, a mapping of the phase space $\boldsymbol{\Omega}$ into $\mathbb{R}^{N}$. Through this mapping, to each point $\mathbf{x}$ there corresponds the point $\mathbf{x}_{s, t}=\boldsymbol{\Theta}(\mathbf{x}, t, s)$ reached at time $s$ by the point which was at $\mathbf{x}$ at the 'initial' time $t$. The flow $\Theta$, restricted to $\boldsymbol{\Lambda}$, has the properties:

Proposition 2.3. Let $\mathrm{x} \in \Omega$ and $t \in \mathbb{R}$ be fixed. Then,

(i) $\Theta(\mathbf{x}, t, t)=\mathbf{x}$.

(ii) $\boldsymbol{\Theta}\left(\mathbf{\Theta}\left(\mathbf{x}, t, s_{1}\right), s_{1}, s_{2}\right)=\boldsymbol{\Theta}\left(\mathbf{x}, t, s_{2}\right), \quad \forall s_{1}, s_{2} \in\left(t-\tau_{-}(\mathbf{x}), t+\tau_{+}(\mathbf{x})\right)$.

(iii) $\boldsymbol{\Theta}(\mathbf{x}, t, s)=\boldsymbol{\Theta}(\mathbf{x}, t-s, 0)=\boldsymbol{\Theta}(\mathbf{x}, 0, s-t), \quad \forall s \in\left(t-\tau_{-}(\mathbf{x}), t+\tau_{+}(\mathbf{x})\right)$.

(iv) $\left|\boldsymbol{\Theta}\left(\mathbf{x}_{1}, t, s\right)-\boldsymbol{\Theta}\left(\mathbf{x}_{2}, t, s\right)\right| \leqslant \exp (\kappa|t-s|)\left|\mathbf{x}_{1}-\mathbf{x}_{2}\right|$ for any $\mathbf{x}_{1}, \mathbf{x}_{2} \in \boldsymbol{\Omega}, s-t \in I_{\mathbf{x}_{1}} \cap I_{\mathbf{x}_{2}}$.

An important consequence of $($ iii $)$ above is that $\boldsymbol{\Theta}(\mathbf{x}, 0, s)=\boldsymbol{\Theta}(\mathbf{x},-s, 0)$ for any $\mathbf{x} \in \boldsymbol{\Omega}$, $0 \leqslant s \leqslant \tau_{+}(\mathbf{x})$. Therefore, from now on, to shorten notations we shall denote

$$
\mathbf{\Phi}(\mathbf{x}, t)=\mathbf{\Theta}(\mathbf{x}, 0, t), \quad \forall t \in \mathbb{R},
$$

so that $\mathbf{\Phi}(\mathbf{x},-t)=\boldsymbol{\Theta}(\mathbf{x}, t, 0), t \in \mathbb{R}$. We define the incoming and outgoing part of the boundary $\partial \boldsymbol{\Omega}$ through the flow $\boldsymbol{\Phi}$ :

Definition 2.4. The incoming $\Gamma_{-}$and the outgoing $\Gamma_{+}$parts of the boundary $\partial \boldsymbol{\Omega}$ are defined by:

$$
\Gamma_{ \pm}:=\left\{\mathbf{y} \in \partial \boldsymbol{\Omega} ; \exists \mathbf{x} \in \boldsymbol{\Omega}, \tau_{ \pm}(\mathbf{x})<\infty \text { and } \mathbf{y}=\mathbf{\Phi}\left(\mathbf{x}, \pm \tau_{ \pm}(\mathbf{x})\right)\right\} .
$$


Properties of $\boldsymbol{\Phi}$ and of $\tau_{ \pm}$imply that $\Gamma_{ \pm}$are Borel sets. It is possible to extend the definition of $\tau_{ \pm}$to $\Gamma_{ \pm}$as follows. If $\mathbf{x} \in \Gamma_{-}$then we put $\tau_{-}(\mathbf{x})=0$ and denote $\tau_{+}(\mathbf{x})$ the length of the integral curve having $\mathbf{x}$ as its left end-point; similarly if $\mathbf{x} \in \Gamma_{+}$then we put $\tau_{+}(\mathbf{x})=0$ and denote $\tau_{-}(\mathbf{x})$ the length of the integral curve having $\mathbf{x}$ as its right endpoint. Note that this definition implies that $\tau_{ \pm}$are measurable over $\Omega \cup \Gamma_{-} \cup \Gamma_{+}$.

Let us illustrate the above definition of $\Gamma_{ \pm}$by two simple $2 D$ examples:

Example 2.5 (Harmonic oscillator in a rectangle). Let $\boldsymbol{\Omega}=(-a, a) \times(-\xi, \xi)$ with $a, \xi>0$ and let us consider the harmonic oscillator force field

$$
\mathscr{F}(\mathbf{x})=\left(v,-\omega^{2} x\right), \quad \text { for any } \mathbf{x}=(x, v) \in \mathbf{\Omega}
$$

where $\omega>0$. We take as $\mu$ the Lebesgue measure over $\mathbb{R}^{2}$ and, since $\mathscr{F}$ is divergence-free, Assumption 1 is fulfilled. In this case, for any $\mathbf{x}_{0}=\left(x_{0}, v_{0}\right) \in \boldsymbol{\Omega}$, the solution $(x(t), v(t))=$ $\mathbf{\Phi}\left(\mathbf{x}_{0}, t\right)$ to the characteristic equation $\frac{\mathrm{d}}{\mathrm{d} t} \mathbf{X}(t)=\mathscr{F}(\mathbf{X}(t)), \mathbf{X}(0)=\mathbf{x}_{0}$, given by

$$
\boldsymbol{\Phi}\left(\mathbf{x}_{0}, t\right)=\left(x_{0} \cos (\omega t)+\frac{v_{0}}{\omega} \sin (\omega t) ;-x_{0} \omega \sin (\omega t)+v_{0} \cos (\omega t)\right)
$$

is such that

$$
\omega^{2} x^{2}(t)+v^{2}(t)=\omega^{2} x_{0}^{2}+v_{0}^{2}, \quad t \in\left(-\tau_{-}\left(\mathbf{x}_{0}\right), \tau_{+}\left(\mathbf{x}_{0}\right)\right)
$$

which means that the integral curves associated to $\mathscr{F}$ are ellipses centered at $(0,0)$ and oriented in the counterclockwise direction. Now,

$$
\partial \boldsymbol{\Omega}=(\{-a\} \times[-\xi, \xi]) \bigcup(\{a\} \times[-\xi, \xi]) \bigcup([-a, a] \times\{-\xi\}) \bigcup([-a, a] \times\{\xi\})
$$

and it is easy to check that

$$
\Gamma_{ \pm}=(\{ \pm a\} \times(-\xi, 0]) \bigcup(\{\mp a\} \times[0, \xi)) \bigcup([0, a) \times\{ \pm \xi\}) \bigcup((-a, 0] \times\{\mp \xi\}) .
$$

Notice that $\Gamma_{+} \cap \Gamma_{-}=\{(a, 0),(0, \xi),(-a, 0),(0,-\xi)\}$ and

$$
\partial \boldsymbol{\Omega} \backslash\left(\Gamma_{+} \cup \Gamma_{-}\right)=\{(a, \xi),(a,-\xi),(-a, \xi),(-a, \xi)\}
$$

is a discrete set (of linear Lebesgue measure zero).

Example 2.6 (Hamonic oscillator in a stadium). Consider now the two-dimensional phase space (where $\mathbb{R}^{2}$ is still endowed with the Lebesgue measure $\mu$ ):

$$
\boldsymbol{\Omega}=\left\{\mathbf{x}=(x, v) \in \mathbb{R}^{2} ; x^{2}+v^{2}<2 \text { and }-1<v<1\right\}
$$

and consider the harmonic oscillator force field $\mathscr{F}$ given by (2.3) with $\omega=1$ for simplicity. Then, the integral curves associated to $\mathscr{F}$ are circles centered at $(0,0)$ and oriented in the counterclockwise direction. In this case, one can see that

$$
\Gamma_{ \pm}=\{(x,-1) ;-1< \pm x \leqslant 0\} \cup\{(x, 1) ; 0 \leqslant \pm x<1\} .
$$

In particular, one sees that $\partial \boldsymbol{\Omega} \backslash\left(\Gamma_{+} \cup \Gamma_{-}\right)=\left\{(x, v) \in \mathbb{R}^{2} ; x^{2}+v^{2}=2 ;-1 \leqslant v \leqslant 1\right\}$ is a 'big' part of the boundary $\partial \boldsymbol{\Omega}$ (with positive linear Lebesgue measure). Notice also that $\tau_{+}(\mathbf{x})=+\infty$ for any $\mathbf{x}=(x, v)$ with $x^{2}+v^{2}<1$.

The main aim of the present discussion is to represent $\Omega$ as a collection of characteristics running between points of $\Gamma_{-}$and $\Gamma_{+}$so that the integral over $\Omega$ can be split into integrals over $\Gamma_{-}\left(\right.$or $\left.\Gamma_{+}\right)$and along the characteristics. However, at present we cannot do this in a precise way since, in general, the sets $\Gamma_{+}$and $\Gamma_{-}$do not provide a partition of $\partial \boldsymbol{\Omega}$ as there may be 'too 
many' characteristics which extend to infinity on either side. Since we have not assumed $\Omega$ to be bounded, $\Gamma_{-}$or $\Gamma_{+}$may be empty and also we may have characteristics running from $-\infty$ to $+\infty$ such as periodic ones. Thus, in general, characteristics starting from $\Gamma_{-}$or ending at $\Gamma_{+}$would not fill the whole $\Omega$ and, to proceed, we have to construct an auxiliary set by extending $\Omega$ into the time domain and use the approach of [9] which is explained below.

2.2. Integration along characteristics. For any $0<T<\infty$, we define the domain

$$
\boldsymbol{\Omega}_{T}=\boldsymbol{\Omega} \times(0, T)
$$

and the measure $\mathrm{d} \mu_{T}=\mathrm{d} \mu \otimes \mathrm{d} t$ on $\Omega_{T}$. Consider the vector field over $\Omega_{T}$ :

$$
Y=\partial_{t}+\mathscr{F}(\mathbf{x}) \cdot \nabla_{\mathbf{x}}=\mathscr{A}(\xi) \cdot \nabla_{\xi}
$$

where $\mathscr{A}(\xi)=(\mathscr{F}(\mathbf{x}), 1)$ for any $\xi=(\mathbf{x}, t)$. We can define the characteristic curves of $\mathscr{A}$ as the solution $\xi(s)=(\mathbf{X}(s), \theta(s))$ to the system $\frac{\mathrm{d}}{\mathrm{d} s} \xi(s)=\mathscr{A}(\xi(s))$, i.e.

$$
\frac{\mathrm{d}}{\mathrm{d} s} \mathbf{X}(s)=\mathscr{F}(\mathbf{X}(s)), \quad \frac{\mathrm{d}}{\mathrm{d} s} \theta(s)=1, \quad(s \in \mathbb{R}),
$$

with

$$
\mathbf{X}(0)=\mathbf{x}, \quad \theta(0)=t .
$$

It is clear that the solution $\xi(s)$ to the above system is given by

$$
\mathbf{X}(s)=\mathbf{\Phi}(\mathbf{x}, s), \quad \theta(s)=s+t,
$$

and we can define the flow of solution $\boldsymbol{\Psi}(\xi, s)=(\mathbf{\Phi}(\mathbf{x}, s), s+t)$ associated to $\mathscr{A}$ and the existence times of the characteristic curves of $Y$ are defined, for any $\xi=(\mathbf{x}, t) \in \boldsymbol{\Omega}_{T}$, as

$$
\ell_{ \pm}(\xi)=\inf \left\{s>0,(\mathbf{\Phi}(\mathbf{x}, \pm s), \pm s+t) \notin \boldsymbol{\Omega}_{T}\right\} .
$$

The flow $\boldsymbol{\Psi}(\cdot, \cdot)$ enjoys, mutatis mutandis, the properties listed in Proposition 2.3 and $\mu_{T}$ is invariant under $\boldsymbol{\Psi}$. Moreover, since $\mathscr{A}$ is clearly Lipschitz continuous on $\overline{\Omega_{T}}$, no characteristic of $Y$ can escape to infinity in finite time. In other words, all characteristic curves of $Y$ now have finite lengths. Indeed, if $\boldsymbol{\Phi}(\mathrm{x}, \pm s)$ does not reach $\partial \boldsymbol{\Omega}$, then the characteristic curve $\boldsymbol{\Psi}(\xi, \pm s)$ enters or leaves $\Omega_{T}$ through the bottom $\Omega \times\{0\}$, or through the top $\Omega \times\{T\}$ of it. Precisely, it is easy to verify that for $\xi=(\mathbf{x}, t) \in \Omega_{T}$ we have

$$
\ell_{+}(\xi)=\tau_{+}(\mathbf{x}) \wedge(T-t) \quad \text { and } \quad \ell_{-}(\xi)=\tau_{-}(\mathbf{x}) \wedge t
$$

where $\wedge$ denotes minimum. This clearly implies $\sup \left\{\ell_{ \pm}(\xi) ; \xi \in \boldsymbol{\Omega}_{T}\right\} \leqslant T$. Define now

$$
\Sigma_{ \pm, T}=\left\{\zeta \in \partial \boldsymbol{\Omega}_{T} ; \exists \xi \in \boldsymbol{\Omega}_{T} \text { such that } \zeta=\boldsymbol{\Psi}\left(\xi, \pm \ell_{ \pm}(\xi)\right)\right\} .
$$

The definition of $\Sigma_{ \pm, T}$ is analogous to $\Gamma_{ \pm}$with the understanding that now the charateristic curves correspond to the vector field $\mathscr{A}$. In other words, $\Sigma_{-, T}$ (resp. $\left.\Sigma_{+, T}\right)$ is the subset of $\partial \boldsymbol{\Omega}_{T}$ consisting of all left (resp. right) limits of characteristic curves of $\mathscr{A}$ in $\boldsymbol{\Omega}_{T}$ whereas $\Gamma_{-}$(resp. $\Gamma_{+}$) is the subset of $\partial \boldsymbol{\Omega}$ consisting of all left (resp. right) limits of characteristic curves of $\mathscr{F}$ in $\boldsymbol{\Omega}$. The main difference (and the interest of such a lifting to $\boldsymbol{\Omega}_{T}$ ) is the fact that each characteristic curve of $\mathscr{A}$ does reach the boundaries $\Sigma_{ \pm, T}$ in finite time. The above formulae allow us to extend functions $\ell_{ \pm}$to $\Sigma_{ \pm, T}$ in the same way as we extended the functions $\tau_{ \pm}$to $\Gamma_{ \pm}$. With these considerations, we can represent, up to a set of zero measure, the phase space $\Omega_{T}$ as

$$
\begin{aligned}
\boldsymbol{\Omega}_{T} & =\left\{\boldsymbol{\Psi}(\xi, s) ; \xi \in \Sigma_{-, T}, 0<s<\ell_{+}(\xi)\right\} \\
& =\left\{\boldsymbol{\Psi}(\xi,-s) ; \xi \in \Sigma_{+, T}, 0<s<\ell_{-}(\xi)\right\} .
\end{aligned}
$$


With this realization we can prove the following:

Proposition 2.7. Let $T>0$ be fixed. There are unique positive Borel measures $\mathrm{d} \nu_{ \pm}$on $\Sigma_{ \pm, T}$ such that $\mathrm{d} \mu_{T}=\mathrm{d} \nu_{+} \otimes \mathrm{d} s=\mathrm{d} \nu_{-} \otimes \mathrm{d} s$.

Proof. For any $\delta>0$, define $\mathscr{E}_{\delta}$ as the set of all bounded Borel subsets $E$ of $\Sigma_{-, T}$ such that $\ell_{+}(\xi)>\delta$ for any $\xi \in E$. Let us now fix $E \in \mathscr{E}_{\delta}$. For all $0<\sigma \leqslant \delta$ put

$$
E_{\sigma}=\{\boldsymbol{\Psi}(\xi, s) ; \xi \in E, 0<s \leqslant \sigma\} .
$$

Clearly $E_{\sigma}$ is a measurable subset of $\boldsymbol{\Omega}_{T}$. Define the mapping $h: \sigma \in(0, \delta] \mapsto h(\sigma)=\mu_{T}\left(E_{\sigma}\right)$ with $h(0)=0$. If $\sigma_{1}$ and $\sigma_{2}$ are two positive numbers such that $\sigma_{1}+\sigma_{2} \leqslant \delta$, then

$$
E_{\sigma_{1}+\sigma_{2}} \backslash E_{\sigma_{1}}=\left\{\boldsymbol{\Psi}(\xi, s) ; \xi \in E, \sigma_{1}<s \leqslant \sigma_{1}+\sigma_{2}\right\}=\left\{\boldsymbol{\Psi}\left(\eta, \sigma_{1}\right) ; \eta \in E_{\sigma_{2}}\right\} .
$$

The properties of the flow $\Psi$ (see Proposition 2.3) ensure that the mapping $\eta \mapsto \Psi\left(\eta, \sigma_{1}\right)$ is one-to-one and measure preserving, so that

$$
\mu_{T}\left(E_{\sigma_{1}+\sigma_{2}} \backslash E_{\sigma_{1}}\right)=\mu_{T}\left(E_{\sigma_{2}}\right)=h\left(\sigma_{2}\right) .
$$

Since $E_{\sigma_{1}+\sigma_{2}}=E_{\sigma_{1}} \cup\left(E_{\sigma_{1}+\sigma_{2}} \backslash E_{\sigma_{1}}\right)$, we immediately obtain

$$
h\left(\sigma_{1}+\sigma_{2}\right)=h\left(\sigma_{1}\right)+h\left(\sigma_{2}\right) \quad \text { for any } \quad \sigma_{1}, \sigma_{2}>0 \text { with } \sigma_{1}+\sigma_{2} \leqslant \delta .
$$

This is the well-known Cauchy equation, though defined only on an interval of the real line. It can be solved in a standard way using non-negativity instead of continuity, yielding:

$$
h(\sigma)=c_{E} \sigma \quad \text { for any } \quad 0<\sigma \leqslant \delta
$$

where $c_{E}=h(\delta) / \delta$. We define $\nu_{-}(E)=c_{E}$. It is not difficult to see that, with the above procedure, the mapping $\nu_{-}(\cdot)$ defines a positive measure on the ring $\mathscr{E}=\bigcup_{\delta>0} \mathscr{E}_{\delta}$ of all the Borel subsets of $\Sigma_{-, T}$ on which the function $\ell_{+}(\xi)$ is bounded away from 0 . Such a measure $\nu_{-}$can be uniquely extended to the $\sigma$-algebra of the Borel subsets of $\Sigma_{-, T}$ (see e.g. [12, Theorem A, p. 54]). Consider now a Borel subset $E$ of $\Sigma_{-, T}$ and a Borel subset $I$ of $\mathbb{R}^{+}$, such that for all $\xi \in E$ and $s \in I$ we have $0<s<\ell_{+}(\xi)$. Then

$$
E \times I=\{\boldsymbol{\Psi}(\xi, s) ; \xi \in E, s \in I\} \subset \boldsymbol{\Omega}_{T} .
$$

Thanks to the definition of $\nu_{-}(\cdot)$, we can state that $\mu_{T}(E \times I)=\nu_{-}(E)$ meas $(I)$ where meas $(I)$ denotes the linear Lebesgue measure of $I \subset \mathbb{R}$. This shows that $\mathrm{d} \mu_{T}=\mathrm{d} \nu_{-} \otimes \mathrm{d} s$. Similarly we can define a measure $\nu_{+}$on $\Sigma_{+, T}$ and prove that $\mathrm{d} \mu_{T}=\mathrm{d} \nu_{+} \otimes \mathrm{d} s$. The uniqueness of the measures $\mathrm{d} \nu_{ \pm}$is then obvious.

Remark 2.8. Note that the above construction of the Borel measures $\mathrm{d} \nu_{ \pm}$differs from that of $[11$, Lemmas XI.3.1 \& 3.2], [9, Propositions $7 \&$ 8] which, moreover, only apply when $\mu$ is absolutely continuous with respect to the Lebesgue measure. Our construction is much more general and can also be generalized to the case of a non-divergence force field $\mathscr{F}$, [5].

Next, by the cylindrical structure of $\boldsymbol{\Omega}_{T}$, and the representation of $\Sigma_{ \pm, T}$ as

$$
\Sigma_{-, T}=\left(\Gamma_{-} \times(0, T)\right) \cup \Omega \times\{0\} \quad \text { and } \quad \Sigma_{+, T}=\left(\Gamma_{+} \times(0, T)\right) \cup \Omega \times\{T\},
$$

the measures $\mathrm{d} \nu_{ \pm}$over $\Gamma_{ \pm} \times(0, T)$ can be written as $\mathrm{d} \nu_{ \pm}=\mathrm{d} \mu_{ \pm} \otimes \mathrm{d} t$, where $\mathrm{d} \mu_{ \pm}$are Borel measures on $\Gamma_{ \pm}$. This leads to the following 
Lemma 2.9. There are unique positive Borel measures $\mathrm{d} \mu_{ \pm}$on $\Gamma_{ \pm}$such that, for any $f \in$ $L^{1}\left(\boldsymbol{\Omega}_{T}, \mathrm{~d} \mu_{T}\right)$

$$
\begin{aligned}
\int_{\boldsymbol{\Omega}_{T}} f(\mathbf{x}, t) \mathrm{d} \mu_{T}(\mathbf{x}, t)= & \int_{0}^{T} \mathrm{~d} t \int_{\Gamma_{+}} \mathrm{d} \mu_{+}(\mathbf{y}) \int_{0}^{\tau_{-}(\mathbf{y}) \wedge t} f(\mathbf{\Phi}(\mathbf{y},-s), t-s) \mathrm{d} s \\
& +\int_{\boldsymbol{\Omega}} \mathrm{d} \mu(\mathbf{x}) \int_{0}^{\tau_{-}(\mathbf{x}) \wedge T} f(\mathbf{\Phi}(\mathbf{x},-s), T-s) \mathrm{d} s,
\end{aligned}
$$

and

$$
\begin{aligned}
\int_{\boldsymbol{\Omega}_{T}} f(\mathbf{x}, t) \mathrm{d} \mu_{T}(\mathbf{x}, t)= & \int_{0}^{T} \mathrm{~d} t \int_{\Gamma_{-}} \mathrm{d} \mu_{-}(\mathbf{y}) \int_{0}^{\tau_{+}(\mathbf{y}) \wedge(T-t)} f(\mathbf{\Phi}(\mathbf{y}, s), t+s) \mathrm{d} s \\
& +\int_{\boldsymbol{\Omega}} \mathrm{d} \mu(\mathbf{x}) \int_{0}^{\tau_{+}(\mathbf{x}) \wedge T} f(\mathbf{\Phi}(\mathbf{x}, s), s) \mathrm{d} s .
\end{aligned}
$$

The above fundamental result allows to compute integrals over the cylindrical phase-space $\boldsymbol{\Omega}_{T}$ through integration along the characteristic curves. Let us now generalize it to the phase space $\Omega$. Here the main difficulty stems from the fact that the characteristic curves of the vector field $\mathscr{F}$ are no longer assumed to be of finite length. In order to extend Lemma 2.9 to possibly infinite existence times, first we prove the following:

Lemma 2.10. Let $T>0$ be fixed. Then, $\tau_{+}(\mathbf{x})<T$ for any $\mathbf{x} \in \mathbf{\Omega}$ if and only if $\tau_{-}(\mathbf{x})<T$ for any $\mathrm{x} \in \boldsymbol{\Omega}$.

Proof. It is easy to see that $\tau_{+}(\mathbf{x})<T$ for any $\mathbf{x} \in \boldsymbol{\Omega}$ is equivalent to $\tau(\mathbf{x})<T$ for any $\mathbf{x} \in \boldsymbol{\Omega}$ and this is also equivalent to $\tau_{-}(\mathbf{x})<T$ for any $\mathbf{x} \in \boldsymbol{\Omega}$.

Hereafter, the support of a measurable function $f$ defined on $\Omega$ is defined as $\operatorname{Supp} f=\Omega \backslash \omega$ where $\omega$ is the maximal open subset of $\Omega$ on which $f$ vanishes $\mathrm{d} \mu$-almost everywhere.

Proposition 2.11. Let $f \in L^{1}(\boldsymbol{\Omega}, \mathrm{d} \mu)$. Assume that there exists $\tau_{0}>0$ such that $\tau_{ \pm}(\mathbf{x})<\tau_{0}$ for any $\mathbf{x} \in \operatorname{Supp}(f)$. Then,

$$
\begin{aligned}
\int_{\boldsymbol{\Omega}} f(\mathbf{x}) \mathrm{d} \mu(\mathbf{x}) & =\int_{\Gamma_{+}} \mathrm{d} \mu_{+}(\mathbf{y}) \int_{0}^{\tau_{-}(\mathbf{y})} f(\mathbf{\Phi}(\mathbf{y},-s)) \mathrm{d} s \\
& =\int_{\Gamma_{-}} \mathrm{d} \mu_{-}(\mathbf{y}) \int_{0}^{\tau_{+}(\mathbf{y})} f(\mathbf{\Phi}(\mathbf{y}, s)) \mathrm{d} s .
\end{aligned}
$$

Proof. For any $T>\tau_{0}$, define the domain $\boldsymbol{\Omega}_{T}=\boldsymbol{\Omega} \times(0, T)$. Since $T<\infty$, it is clear that $f \in L^{1}\left(\boldsymbol{\Omega}_{T}, \mathrm{~d} \mu \mathrm{d} t\right)$ and, by (2.6), we get

$$
\begin{aligned}
T \int_{\Omega} f(\mathbf{x}) \mathrm{d} \mu(\mathbf{x})=\int_{0}^{T} \mathrm{~d} t \int_{\Gamma_{+}} \mathrm{d} \mu_{+}(\mathbf{y}) \int_{0}^{t \wedge \tau_{-}(\mathbf{y})} f(\mathbf{\Phi}(\mathbf{y},-s)) \mathrm{d} s+ \\
\qquad \int_{\Omega} \mathrm{d} \mu(\mathbf{x}) \int_{0}^{\tau_{-}(\mathbf{x})} f(\mathbf{\Phi}(\mathbf{x},-s)) \mathrm{d} s .
\end{aligned}
$$

Since the formula is valid for any $T>\tau_{0}$, differentiating with respect to $T$ leads to the first assertion. The second assertion is proved in the same way by using formula (2.7). 
To drop the finiteness assumption on $\tau_{ \pm}(\mathbf{x})$, first we introduce the sets

$$
\boldsymbol{\Omega}_{ \pm}=\left\{\mathbf{x} \in \boldsymbol{\Omega} ; \tau_{ \pm}(\mathrm{x})<\infty\right\}, \quad \boldsymbol{\Omega}_{ \pm \infty}=\left\{\mathrm{x} \in \boldsymbol{\Omega} ; \tau_{ \pm}(\mathrm{x})=\infty\right\},
$$

and

$$
\Gamma_{ \pm \infty}=\left\{\mathbf{y} \in \Gamma_{ \pm} ; \tau_{\mp}(\mathbf{y})=\infty\right\}
$$

Then

Proposition 2.12. Given $f \in L^{1}(\boldsymbol{\Omega}, \mathrm{d} \mu)$, one has

$$
\int_{\boldsymbol{\Omega}_{ \pm}} f(\mathbf{x}) \mathrm{d} \mu(\mathbf{x})=\int_{\Gamma_{ \pm}} \mathrm{d} \mu_{ \pm}(\mathbf{y}) \int_{0}^{\tau_{\mp}(\mathbf{y})} f(\mathbf{\Phi}(\mathbf{y}, \mp s)) \mathrm{d} s,
$$

and

$$
\int_{\boldsymbol{\Omega}_{ \pm} \cap \boldsymbol{\Omega}_{\mp \infty}} f(\mathbf{x}) \mathrm{d} \mu(\mathbf{x})=\int_{\Gamma_{ \pm} \infty} \mathrm{d} \mu_{ \pm}(\mathbf{y}) \int_{0}^{\infty} f(\mathbf{\Phi}(\mathbf{y}, \mp s)) \mathrm{d} s .
$$

Proof. Assume first $f \geqslant 0$. Let us fix $T>0$. It is clear that $\mathbf{x} \in \boldsymbol{\Omega}$ satisfies $\tau_{+}(\mathbf{x})<T$ if and only if $\mathbf{x}=\boldsymbol{\Phi}(\mathbf{y},-s)$, with $\mathbf{y} \in \Gamma_{+}$and $0<s<T \wedge \tau_{-}(\mathbf{y})$. Then, by Proposition 2.11,

$$
\int_{\left\{\tau_{+}(\mathbf{x})<T\right\}} f(\mathbf{x}) \mathrm{d} \mu(\mathbf{x})=\int_{\Gamma_{+}} \mathrm{d} \mu_{+}(\mathbf{y}) \int_{0}^{T \wedge \tau_{-}(\mathbf{y})} f(\boldsymbol{\Phi}(\mathbf{y},-s)) \mathrm{d} s .
$$

Since $f \geqslant 0$, the inner integral is increasing with $T$ and, using the monotone convergence theorem, we let $T \rightarrow \infty$ to get

$$
\int_{\boldsymbol{\Omega}_{+}} f(\mathbf{x}) \mathrm{d} \mu(\mathbf{x})=\int_{\Gamma_{+}} \mathrm{d} \mu_{+}(\mathbf{y}) \int_{0}^{\tau_{-}(\mathbf{y})} f(\boldsymbol{\Phi}(\mathbf{y},-s)) \mathrm{d} s
$$

which coincides with (2.9). We proceed in the same way with integration on $\Gamma_{-}$and get the second part of (2.9). Next we consider the set

$$
\Delta=\left\{\mathbf{x} \in \mathbf{\Omega} ; \mathbf{x}=\mathbf{\Phi}(\mathbf{y},-s), \mathbf{y} \in \boldsymbol{\Omega}_{+\infty}, 0<s<T\right\} .
$$

Proposition 2.11 asserts that

$$
\int_{\Delta} f(\mathbf{x}) \mathrm{d} \mu(\mathbf{x})=\int_{\boldsymbol{\Omega}_{+\infty}} \mathrm{d} \mu_{+}(\mathbf{y}) \int_{0}^{T} f(\mathbf{\Phi}(\mathbf{y},-s)) \mathrm{d} s .
$$

Letting again $T \rightarrow \infty$, we get (2.10). We extend the results to arbitrary $f$ by linearity.

Finally, with the following, we show that it is possible to transfer integrals over $\Gamma_{-}$to $\Gamma_{+}$:

Proposition 2.13. For any $\psi \in L^{1}\left(\Gamma_{-}, \mathrm{d} \mu_{-}\right)$,

$$
\int_{\Gamma_{-} \backslash \Gamma_{-} \infty} \psi(\mathbf{y}) \mathrm{d} \mu_{-}(\mathbf{y})=\int_{\Gamma_{+} \backslash \Gamma_{+} \infty} \psi\left(\mathbf{\Phi}\left(\mathbf{z},-\tau_{-}(\mathbf{z})\right)\right) \mathrm{d} \mu_{+}(\mathbf{z}) .
$$

Proof. For any $\epsilon>0$, let $f_{\epsilon}$ be the function defined on $\boldsymbol{\Omega}_{+} \cap \boldsymbol{\Omega}_{-}$by

$$
\psi_{\epsilon}(\mathbf{x})= \begin{cases}\frac{\psi\left(\mathbf{\Phi}\left(\mathbf{x},-\tau_{-}(\mathbf{x})\right)\right)}{\tau_{+}(\mathbf{x})+\tau_{-}(\mathbf{x})} & \text { if } \tau_{-}(\mathbf{x})+\tau_{+}(\mathbf{x})>\epsilon, \\ 0 & \text { else. }\end{cases}
$$


Since $\psi_{\epsilon} \in L^{1}\left(\boldsymbol{\Omega}_{+} \cap \boldsymbol{\Omega}_{-}, \mathrm{d} \mu\right)$, Eqs. (2.9) and (2.10) give

$$
\begin{aligned}
\int_{\boldsymbol{\Omega}_{+} \cap \boldsymbol{\Omega}_{-}} \psi_{\epsilon}(\mathbf{x}) \mathrm{d} \mu(\mathbf{x}) & =\int_{\left\{\tau_{+}(\mathbf{y})>\epsilon\right\} \backslash \Gamma_{-}} \mathrm{d} \mu_{-}(\mathbf{y}) \int_{0}^{\tau_{+}(\mathbf{y})} \psi(\mathbf{y}) \frac{\mathrm{d} s}{\tau_{+}(\mathbf{y})} \\
& =\int_{\left\{\tau_{+}(\mathbf{y})>\epsilon\right\} \backslash \Gamma_{-\infty}} \psi(\mathbf{y}) \mathrm{d} \mu_{-}(\mathbf{y}) .
\end{aligned}
$$

In the same way,

$$
\begin{aligned}
\int_{\boldsymbol{\Omega}_{+} \cap \boldsymbol{\Omega}_{-}} \psi_{\epsilon}(\mathbf{x}) \mathrm{d} \mu(\mathbf{x}) & =\int_{\left\{\tau_{-}(\mathbf{y})>\epsilon\right\} \backslash \Gamma_{+\infty}} \mathrm{d} \mu_{+}(\mathbf{y}) \int_{0}^{\tau_{-}(\mathbf{y})} \psi\left(\mathbf{\Phi}\left(\mathbf{y},-\tau_{-}(\mathbf{y})\right)\right) \frac{\mathrm{d} s}{\tau_{-}(\mathbf{y})} \\
& =\int_{\left\{\tau_{-}(\mathbf{y})>\epsilon\right\} \backslash \Gamma_{+\infty}} \psi\left(\mathbf{\Phi}\left(\mathbf{y},-\tau_{-}(\mathbf{y})\right)\right) \mathrm{d} \mu_{-}(\mathbf{y}),
\end{aligned}
$$

which leads to

$$
\int_{\left\{\tau_{-}(\mathbf{y})>\epsilon\right\} \backslash \Gamma_{+\infty}} \psi\left(\mathbf{\Phi}\left(\mathbf{y},-\tau_{-}(\mathbf{y})\right)\right) \mathrm{d} \mu_{+}(\mathbf{y})=\int_{\left\{\tau_{+}(\mathbf{y})>\epsilon\right\} \backslash \Gamma_{-\infty}} \psi(\mathbf{y}) \mathrm{d} \mu_{-}(\mathbf{y})
$$

for any $\epsilon>0$. Passing to the limit as $\epsilon \rightarrow 0$ we get the conclusion.

We end this section with a technical result we shall need in the sequel (see Lemma 3.3):

Proposition 2.14. Let $K$ be a compact subset of $\Omega$. Denote

$$
K_{ \pm}:=\left\{\mathbf{y} \in \Gamma_{ \pm} ; \exists t_{0} \in \mathbb{R} \text { such that } \mathbf{\Phi}(\mathbf{y}, \pm t) \in K \text { for any } t \geqslant t_{0}\right\} .
$$

Then $\mu_{ \pm}\left(K_{ \pm}\right)=0$.

Proof. Let $K$ be a fixed compact subset of $\Omega$. Applying Eq. (2.9) or (2.10) to the function $f(\mathbf{x})=\chi_{K}(\mathbf{x})$, one has

$$
\infty>\mu(K) \geqslant \int_{K_{-}} \mathrm{d} \mu_{-}(\mathbf{y}) \int_{0}^{\infty} \chi_{K}(\mathbf{\Phi}(\mathbf{y}, t)) \mathrm{d} t .
$$

By definition, if $\mathbf{y} \in K_{-}$, then for some $t_{0} \in \mathbb{R}, \chi_{K}(\mathbf{\Phi}(\mathbf{y}, t))=1$ for any $t \geqslant t_{0}$. Therefore,

$$
\int_{0}^{\infty} \chi_{K}(\mathbf{\Phi}(\mathbf{y}, t))=\infty, \quad \forall \mathbf{y} \in K_{-} .
$$

Inequality (2.12) implies that $\mu_{-}\left(K_{-}\right)=0$. One proves the result for $K_{+}$in the same way.

\section{THE MAXIMAL TRANSPORT OPERATOR AND TRACE RESULTS}

The results of the previous section allow us to define the (maximal) transport operator $\mathcal{T}_{\max }$ as the weak derivative along the characteristic curves. To be precise, let us define the space of test functions $\mathfrak{Y}$ as follows:

Definition 3.1 (Test-functions). Let $\mathfrak{Y}$ be the set of all measurable and bounded functions $\psi$ : $\Omega \rightarrow \mathbb{R}$ with compact support in $\Omega$ and such that, for any $\mathrm{x} \in \boldsymbol{\Omega}$, the mapping

$$
s \in\left(-\tau_{-}(\mathbf{x}), \tau_{+}(\mathbf{x})\right) \longmapsto \psi(\mathbf{\Phi}(\mathbf{x}, s))
$$

is continuously differentiable with

$$
\left.\mathbf{x} \in \boldsymbol{\Omega} \longmapsto \frac{\mathrm{d}}{\mathrm{d} s} \psi(\mathbf{\Phi}(\mathbf{x}, s))\right|_{s=0} \text { measurable and bounded } .
$$


Remark 3.2. Notice that the class of test-functions $\mathfrak{Y}$ is not defined as a subset of $L^{\infty}(\Omega, \mathrm{d} \mu)$; that is, we do not identify functions equal $\mu$-almost everywhere. It is however a natural question to know whether two test-functions coinciding $\mu$-almost everywhere are such that there derivatives (defined by (3.1)) do coincide $\mu$-almost everywhere. We provide a positive answer to this question at the end of the paper (see Appendix).

An important property of test-functions is the following consequence of Proposition 2.14:

Lemma 3.3. Let $\psi \in \mathfrak{Y}$ be given. For $\mu_{\mp}$-almost any $\mathbf{y} \in \Gamma_{\mp}$ there exists a sequence $\left(t_{n}^{ \pm}\right)_{n}$ (depending on $\mathbf{y}$ ) such that

$$
\lim _{n \rightarrow \infty} t_{n}^{ \pm}=\tau_{ \pm}(\mathbf{y}) \quad \text { and } \quad \psi\left(\mathbf{\Phi}\left(\mathbf{y}, \pm t_{n}^{ \pm}\right)\right)=0 \quad \forall n \in \mathbb{N} .
$$

Proof. Let $\psi \in \mathfrak{Y}$ be given and let $K=\operatorname{Supp}(\psi)$. For any $\mathbf{y} \in \Gamma_{-}$with $\tau_{+}(\mathbf{y})<\infty$ one has $\boldsymbol{\Phi}\left(\mathbf{y}, \tau_{+}(\mathbf{y})\right) \in \Gamma_{+}$and, since $K$ is compact in $\boldsymbol{\Omega}, \psi\left(\mathbf{\Phi}\left(\mathbf{y}, \tau_{+}(\mathbf{y})\right)=0\right.$ and the existence of a sequence $\left(t_{n}^{+}\right)_{n}$ converging to $\tau_{+}(\mathbf{y})$ with the above property is clear. Now, Proposition 2.14 applied to $K$ shows that there exists a set $\Gamma_{-}^{\prime} \subset \Gamma_{-}$with $\mu_{-}\left(\Gamma \backslash \Gamma_{-}^{\prime}\right)=0$ and such that, for any $\mathbf{y} \in \Gamma_{-}^{\prime}$, there is a sequence $\left(t_{n}^{+}\right)_{n}$ converging to $\infty$ such that $\mathbf{\Phi}\left(\mathbf{y}, t_{n}\right) \notin K$ for any $n \in \mathbb{N}$. This proves the result. The statement for $\Gamma_{+}$is proved in the same way.

In the next step we define the transport operator $\left(\mathcal{T}_{\max }, \mathscr{D}\left(\mathcal{T}_{\max }\right)\right)$.

Definition 3.4 (Transport operator $\left.\mathcal{T}_{\max }\right)$. The domain of the maximal transport operator $\mathcal{T}_{\max }$ is the set $\mathscr{D}\left(\mathcal{T}_{\max }\right)$ of all $f \in L^{1}(\Omega, \mathrm{d} \mu)$ for which there exists $g \in L^{1}(\Omega, \mathrm{d} \mu)$ such that

$$
\int_{\boldsymbol{\Omega}} g(\mathbf{x}) \psi(\mathbf{x}) \mathrm{d} \mu(\mathbf{x})=\left.\int_{\boldsymbol{\Omega}} f(\mathbf{x}) \frac{\mathrm{d}}{\mathrm{d} s} \psi(\mathbf{\Phi}(\mathbf{x}, s))\right|_{s=0} \mathrm{~d} \mu(\mathbf{x}), \quad \forall \psi \in \mathfrak{Y} .
$$

In this case, $g=: \mathcal{T}_{\max } f$.

Remark 3.5. Of course, in some weak sense, $\mathcal{T}_{\max } f=-\mathscr{F} \cdot \nabla f$. Precisely, for any $\varphi \in \mathscr{C}_{0}^{1}(\boldsymbol{\Omega})$, the following formula holds:

$$
\int_{\Omega}(\mathscr{F}(\mathbf{x}) \cdot \nabla \varphi(\mathbf{x})) f(\mathbf{x}) \mathrm{d} \mu(\mathbf{x})=\int_{\Omega} \mathcal{T}_{\max } f(\mathbf{x}) \varphi(\mathbf{x}) \mathrm{d} \mu(\mathbf{x}) .
$$

3.1. Fundamental representation formula: mild formulation. Recall that, if $f_{1}$ and $f_{2}$ are two functions defined over $\Omega$, we say that $f_{2}$ is a representative of $f_{1}$ if $\mu\left\{\mathbf{x} \in \Omega ; f_{1}(\mathbf{x}) \neq f_{2}(\mathbf{x})\right\}=$ 0 , i.e. when $f_{1}(\mathbf{x})=f_{2}(\mathbf{x})$ for $\mu$-almost every $\mathbf{x} \in \Omega$. The following fundamental result provides a characterization of the domain of $\mathscr{D}\left(\mathcal{T}_{\max }\right)$ :

Theorem 3.6. Let $f \in L^{1}(\boldsymbol{\Omega}, \mu)$. The following are equivalent:

(1) There exists $g \in L^{1}(\Omega, \mu)$ and a representative $f^{\sharp}$ of $f$ such that, for $\mu$-almost every $\mathbf{x} \in \mathbf{\Omega}$ and any $-\tau_{-}(\mathbf{x})<t_{1} \leqslant t_{2}<\tau_{+}(\mathbf{x}):$

$$
f^{\sharp}\left(\mathbf{\Phi}\left(\mathbf{x}, t_{1}\right)\right)-f^{\sharp}\left(\mathbf{\Phi}\left(\mathbf{x}, t_{2}\right)\right)=\int_{t_{1}}^{t_{2}} g(\boldsymbol{\Phi}(\mathbf{x}, s)) \mathrm{d} s .
$$

(2) $f \in \mathscr{D}\left(\mathcal{T}_{\max }\right)$. In this case, $g=\mathcal{T}_{\max } f$.

The proof of the theorem is made of several steps. The difficult part of the proof is the implication $(2) \Longrightarrow(1)$. It is carried out through several technical lemmas based upon mollification along the characteristic curves (recall that, whenever $\mu$ is not absolutely continuous with respect to the Lebesgue measure, no global convolution argument is available). Let us make precise what 
this is all about. Consider a sequence $\left(\varrho_{n}\right)_{n}$ of one dimensional mollifiers supported in [0,1], i.e. for any $n \in \mathbb{N}, \varrho_{n} \in \mathscr{C}_{0}^{\infty}(\mathbb{R}), \varrho_{n}(s)=0$ if $s \notin[0,1 / n], \varrho_{n}(s) \geqslant 0$ and $\int_{0}^{1 / n} \varrho_{n}(s) \mathrm{d} s=1$. Then, for any $f \in L^{1}(\Omega, \mathrm{d} \mu)$, define the (extended) mollification:

$$
\varrho_{n} \diamond f(\mathbf{x})=\int_{0}^{\tau_{-}(\mathbf{x})} \varrho_{n}(s) f(\mathbf{\Phi}(\mathbf{x},-s)) \mathrm{d} s .
$$

As we shall see later, such a definition corresponds precisely to a time convolution over any characteristic curves (see e.g. (3.4)). Note that, with such a definition, it is not clear a priori that $\varrho_{n} \diamond f$ defines a measurable function, finite almost everywhere. It is proved in the following that actually such a function is integrable.

Lemma 3.7. Given $f \in L^{1}(\boldsymbol{\Omega}, \mathrm{d} \mu), \varrho_{n} \diamond f \in L^{1}(\boldsymbol{\Omega}, \mathrm{d} \mu)$ for any $n \in \mathbb{N}$. Moreover,

$$
\left\|\varrho_{n} \diamond f\right\| \leqslant\|f\|, \quad \forall f \in L^{1}(\boldsymbol{\Omega}, \mathrm{d} \mu), n \in \mathbb{N} .
$$

Proof. One considers, for a given $f \in L^{1}(\Omega, \mathrm{d} \mu)$, the extension of $f$ by zero outside $\Omega$ :

$$
\bar{f}(\mathbf{x})=f(\mathbf{x}), \quad \forall \mathbf{x} \in \mathbf{\Omega}, \quad \bar{f}(\mathbf{x})=0 \quad \forall \mathbf{x} \in \mathbb{R}^{N} \backslash \boldsymbol{\Omega} .
$$

Then $\bar{f} \in L^{1}\left(\mathbb{R}^{N}, \mathrm{~d} \mu\right)$. Let us consider the transformation:

$$
\Upsilon:(\mathbf{x}, s) \in \mathbb{R}^{N} \times \mathbb{R} \mapsto \Upsilon(\mathbf{x}, s)=(\mathbf{\Phi}(\mathbf{x},-s),-s) \in \mathbb{R}^{N} \times \mathbb{R} .
$$

As a homeomorphism, $\Upsilon$ is measure preserving for pure Borel measures. It is also measure preserving for completions of Borel measures (such as a Lebesgue measure) since it is measurepreserving on Borel sets and the completion of a measure is obtained by adding to the Borel $\sigma$-algebra all sets contained in a measure-zero Borel sets, see [12, Theorem 13.B, p. 55]. Then, according to [12, Theorem 39.B, p. 162], the mapping

$$
(\mathbf{x}, s) \in \mathbb{R}^{N} \times \mathbb{R} \mapsto \bar{f}(\mathbf{\Phi}(\mathbf{x},-s))
$$

is measurable as the composition of $\Upsilon$ with the measurable function $(\mathbf{x}, s) \mapsto \bar{f}(\mathbf{x})$. Define now $\Lambda=\left\{(\mathbf{x}, s) ; \mathbf{x} \in \boldsymbol{\Omega}, 0<s<\tau_{-}(\mathbf{x})\right\}, \Lambda$ is a measurable subset of $\mathbb{R}^{N} \times \mathbb{R}$. Therefore, the mapping

$$
(\mathbf{x}, s) \in \mathbb{R}^{N} \times \mathbb{R} \longmapsto \bar{f}(\mathbf{\Phi}(\mathbf{x},-s)) \chi_{\Lambda}(\mathbf{x}, s) \varrho_{n}(s)
$$

is measurable. Since $\varrho_{n}$ is compactly supported, it is also integrable over $\mathbb{R}^{N} \times \mathbb{R}$ and, according to Fubini's Theorem

$$
\left[\varrho_{n} \diamond f\right](\mathbf{x}):=\int_{\mathbb{R}} \bar{f}(\mathbf{\Phi}(\mathbf{x},-s)) \chi_{\Lambda}(\mathbf{x}, s) \varrho_{n}(s) \mathrm{d} s=\int_{0}^{\tau_{-}(\mathbf{x})} \varrho_{n}(s) f(\mathbf{\Phi}(\mathbf{x},-s)) \mathrm{d} s
$$

is finite for almost every $\mathbf{x} \in \Omega$ the and the associated application $\varrho_{n} \diamond f$ is integrable.

Let us prove now (3.3). Since $\left|\varrho_{n} \diamond f\right| \leqslant \varrho_{n} \diamond|f|$, to show that $\varrho_{n} \diamond f \in L^{1}(\boldsymbol{\Omega}, \mathrm{d} \mu)$, it suffices to deal with a nonnegative function $f \in L^{1}(\boldsymbol{\Omega}, \mathrm{d} \mu)$. One sees easily that, for any $\mathbf{y} \in \Gamma_{-}$and any $0<t<\tau_{+}(\mathbf{y})$

$$
\left(\varrho_{n} \diamond f\right)(\mathbf{\Phi}(\mathbf{y}, t))=\int_{0}^{t} \varrho_{n}(s) f(\mathbf{\Phi}(\mathbf{y}, t-s)) \mathrm{d} s=\int_{0}^{t} \varrho_{n}(t-s) f(\mathbf{\Phi}(\mathbf{y}, s)) \mathrm{d} s .
$$


Thus,

$$
\begin{aligned}
\int_{0}^{\tau_{+}(\mathbf{y})}\left[\varrho_{n} \diamond f\right](\boldsymbol{\Phi}(\mathbf{y}, t)) \mathrm{d} t & =\int_{0}^{\tau_{+}(\mathbf{y})} \mathrm{d} s \int_{s}^{\tau_{+}(\mathbf{y})} \varrho_{n}(s) f(\mathbf{\Phi}(\mathbf{y}, t-s)) \mathrm{d} t \\
& =\int_{0}^{\tau_{+}(\mathbf{y}) \wedge 1 / n} \varrho_{n}(s) \mathrm{d} s \int_{0}^{\tau_{+}(\mathbf{y})-s} f(\mathbf{\Phi}(\mathbf{y}, r)) \mathrm{d} r .
\end{aligned}
$$

Therefore,

$$
\begin{aligned}
0 \leqslant \int_{0}^{\tau_{+}(\mathbf{y})}\left[\varrho_{n} \diamond f\right](\mathbf{\Phi}(\mathbf{y}, t)) \mathrm{d} t & \leqslant \int_{0}^{1 / n} \varrho_{n}(s) \mathrm{d} s \int_{0}^{\tau_{+}(\mathbf{y})} f(\mathbf{\Phi}(\mathbf{y}, r)) \mathrm{d} r \\
& =\int_{0}^{\tau_{+}(\mathbf{y})} f(\mathbf{\Phi}(\mathbf{y}, r)) \mathrm{d} r, \quad \forall \mathbf{y} \in \Gamma_{-}, n \in \mathbb{N}
\end{aligned}
$$

so that

$$
\int_{\Gamma_{-}} \mathrm{d} \mu_{-}(\mathbf{y}) \int_{0}^{\tau_{+}(\mathbf{y})}\left[\varrho_{n} \diamond f\right](\mathbf{\Phi}(\mathbf{y}, t)) \mathrm{d} t \leqslant \int_{\Gamma_{-}} \mathrm{d} \mu_{-}(\mathbf{y}) \int_{0}^{\tau_{+}(\mathbf{y})} f(\boldsymbol{\Phi}(\mathbf{y}, r)) \mathrm{d} r .
$$

This proves, thanks to Proposition 2.12, that

$$
\int_{\Omega_{-}}\left[\varrho_{n} \diamond f\right] \mathrm{d} \mu \leqslant \int_{\Omega_{-}} f \mathrm{~d} \mu
$$

Now, in the same way:

$$
\begin{aligned}
\int_{\boldsymbol{\Omega}_{+} \cap \boldsymbol{\Omega}_{-\infty}} & {\left[\varrho_{n} \diamond f\right](\mathbf{x}) \mathrm{d} \mu(\mathbf{x})=\int_{\Gamma_{+\infty}} \mathrm{d} \mu_{+}(\mathbf{y}) \int_{0}^{\infty}\left[\varrho_{n} \diamond f\right](\mathbf{\Phi}(\mathbf{y},-t)) \mathrm{d} t } \\
& =\int_{\Gamma_{+\infty}} \mathrm{d} \mu_{+}(\mathbf{y}) \int_{0}^{\infty} \mathrm{d} t \int_{0}^{\infty} \varrho_{n}(s) f(\mathbf{\Phi}(\mathbf{y},-s-t)) \mathrm{d} s \\
& =\int_{\Gamma_{+\infty}} \mathrm{d} \mu_{+}(\mathbf{y}) \int_{0}^{\infty} \mathrm{d} t \int_{t}^{\infty} \varrho_{n}(r-t) f(\mathbf{\Phi}(\mathbf{y},-r)) \mathrm{d} r .
\end{aligned}
$$

so that

$$
\begin{aligned}
\int_{\boldsymbol{\Omega}_{+} \cap \boldsymbol{\Omega}_{-\infty}}\left[\varrho_{n} \diamond f\right](\mathbf{x}) \mathrm{d} \mu(\mathbf{x}) & =\int_{\Gamma_{+\infty}} \mathrm{d} \mu_{+}(\mathbf{y}) \int_{0}^{\infty} f(\mathbf{\Phi}(\mathbf{y},-r)) \mathrm{d} r \int_{0}^{r} \varrho_{n}(r-t) \mathrm{d} t \\
& \leqslant \int_{\Gamma_{+\infty}} \mathrm{d} \mu_{+}(\mathbf{y}) \int_{0}^{\infty} f(\boldsymbol{\Phi}(\mathbf{y},-r)) \mathrm{d} r
\end{aligned}
$$

i.e.

Finally

$$
\int_{\boldsymbol{\Omega}_{+} \cap \boldsymbol{\Omega}_{-\infty}} \varrho_{n} \diamond f(\mathbf{x}) \mathrm{d} \mu(\mathbf{x}) \leqslant \int_{\boldsymbol{\Omega}_{+} \cap \boldsymbol{\Omega}_{-\infty}} f(\mathbf{x}) \mathrm{d} \mu(\mathbf{x}) .
$$

$$
\begin{aligned}
\int_{\boldsymbol{\Omega}_{+\infty} \cap \boldsymbol{\Omega}_{-\infty}}\left[\varrho_{n} \diamond f\right](\mathbf{x}) \mathrm{d} \mu(\mathbf{x}) & =\int_{\boldsymbol{\Omega}_{+\infty} \cap \boldsymbol{\Omega}_{-\infty}} \mathrm{d} \mu(\mathbf{x}) \int_{0}^{\infty} \varrho_{n}(s) f(\mathbf{\Phi}(\mathbf{x},-s)) \mathrm{d} s \\
& =\int_{0}^{\infty} \varrho_{n}(s) \mathrm{d} s \int_{\boldsymbol{\Omega}_{+\infty} \cap \boldsymbol{\Omega}_{-\infty}} f(\mathbf{\Phi}(\mathbf{x},-s)) \mathrm{d} \mu(\mathbf{x}) .
\end{aligned}
$$

Now, from Assumption 1, for any $s \geqslant 0$,

$$
\int_{\boldsymbol{\Omega}_{+\infty} \cap \boldsymbol{\Omega}_{-\infty}} f(\boldsymbol{\Phi}(\mathbf{x},-s)) \mathrm{d} \mu(\mathbf{x})=\int_{\boldsymbol{\Omega}_{+\infty} \cap \boldsymbol{\Omega}_{-\infty}} f(\mathbf{x}) \mathrm{d} \mu(\mathbf{x}),
$$


so that

$$
\int_{\boldsymbol{\Omega}_{+\infty} \cap \boldsymbol{\Omega}_{-\infty}}\left[\varrho_{n} \diamond f\right](\mathbf{x}) \mathrm{d} \mu(\mathbf{x})=\int_{\boldsymbol{\Omega}_{+\infty} \cap \boldsymbol{\Omega}_{-\infty}} f(\mathbf{x}) \mathrm{d} \mu(\mathbf{x}) .
$$

Combining (3.5), (3.6) and (3.7), one finally gets $\left\|\varrho_{n} \diamond f\right\| \leqslant\|f\|$.

As it is the case for classical convolution, the family $\left(\varrho_{n} \diamond f\right)_{n}$ approximates $f$ in $L^{1}$-norm:

Proposition 3.8. Given $f \in L^{1}(\boldsymbol{\Omega}, \mathrm{d} \mu)$,

$$
\lim _{n \rightarrow \infty} \int_{\Omega}\left|\left(\varrho_{n} \diamond f\right)(\mathbf{x})-f(\mathbf{x})\right| \mathrm{d} \mu(\mathbf{x})=0 .
$$

Proof. According to (3.3) and from the density of $\mathscr{C}_{0}(\boldsymbol{\Omega})$ in $L^{1}(\Omega, \mathrm{d} \mu)$, it suffices to prove the result for any $f$ continuous over $\Omega$ and compactly supported. Splitting $f$ into positive and negative parts, $f=f^{+}-f^{-}$, one can also assume $f$ to be nonnegative. From the continuity of both $f$ and $\Phi(\cdot, \cdot)$, one has

$$
\mathscr{K}_{n}:=\operatorname{Supp}\left(\varrho_{n} \diamond f\right)=\overline{\left\{\mathbf{x} \in \boldsymbol{\Omega}, \exists s_{0} \in \operatorname{Supp}\left(\varrho_{n}\right) \text { such that } \mathbf{\Phi}\left(\mathbf{x},-s_{0}\right) \in \operatorname{Supp}(f)\right\}} .
$$

Moreover, it is easily seen that $\mathscr{K}_{n+1} \subset \mathscr{K}_{n}$ for any $n \geqslant 1$. Finally, it is clear that

$$
\mathscr{K}_{1} \subset\{\mathbf{x} \in \bar{\Omega} ; \exists \mathbf{y} \in \operatorname{Supp}(f) \text { with }|\mathbf{x}-\mathbf{y}| \leqslant d\}
$$

where $d=\sup \{|\boldsymbol{\Phi}(\mathbf{x}, s)-\mathbf{x}| ; 0 \leqslant s \leqslant 1 ; \mathbf{x} \in \operatorname{Supp}(f)\}<\infty$. Therefore, $\mathscr{K}_{1}$ is compact. Set now

$$
\mathcal{O}_{n}:=\mathscr{K}_{n} \cup \operatorname{Supp}(f) \quad \text { and } \quad \mathcal{O}_{n}^{-}=\left\{\mathbf{x} \in \mathcal{O}_{n} ; \tau_{-}(\mathbf{x})<1 / n\right\} .
$$

Noticing that $\mu\left(\mathcal{O}_{1}\right)$ is finite, one can see easily that $\lim _{n} \mu\left(\mathcal{O}_{n}^{-}\right)=0$. Since $\sup _{\mathbf{x} \in \boldsymbol{\Omega}}\left|\varrho_{n} \diamond f(\mathbf{x})\right| \leqslant$ $\sup _{\mathbf{x} \in \mathbf{\Omega}}|f(\mathbf{x})|$, for any $\varepsilon>0$, there exists $n_{0} \geqslant 1$ such that

$$
\int_{\mathcal{O}_{n}^{-}}|f(\mathbf{x})| \mathrm{d} \mu(\mathbf{x}) \leqslant \varepsilon, \quad \text { and } \quad \int_{\mathcal{O}_{n}^{-}}\left|\varrho_{n} \diamond f(\mathbf{x})\right| \mathrm{d} \mu(\mathbf{x}) \leqslant \varepsilon \quad \forall n \geqslant n_{0} .
$$

Now, noticing that $\operatorname{Supp}\left(\varrho_{n} \diamond f-f\right) \subset \mathcal{O}_{n}$, one has for any $n \geqslant n_{0}$,

$$
\int_{\Omega}\left|\varrho_{n} \diamond f-f\right| \mathrm{d} \mu=\int_{\mathcal{O}_{n}}\left|\varrho_{n} \diamond f-f\right| \leqslant 2 \varepsilon+\int_{\mathcal{O}_{n} \backslash \mathcal{O}_{n}^{-}}\left|\varrho_{n} \diamond f-f\right| \mathrm{d} \mu .
$$

For any $\mathbf{x} \in \mathcal{O}_{n} \backslash \mathcal{O}_{n}^{-}$, since $\varrho$ is supported in $[0,1 / n]$, one has

$$
\begin{aligned}
{\left[\varrho_{n} \diamond f\right](\mathbf{x})-f(\mathbf{x}) } & =\int_{0}^{1 / n} \varrho_{n}(s) f(\boldsymbol{\Phi}(\mathbf{x},-s)) \mathrm{d} s-f(\mathbf{x}) \\
& =\int_{0}^{1 / n} \varrho_{n}(s)(f(\mathbf{\Phi}(\mathbf{x},-s))-f(\mathbf{x})) \mathrm{d} s .
\end{aligned}
$$

Note that, thanks to Gronwall's lemma,

$$
|\mathbf{\Phi}(\mathbf{x},-s)-\mathbf{x}| \leqslant \frac{L}{\kappa}(\exp (k s)-1) \leqslant \frac{L}{\kappa}(\exp (\kappa / n)-1), \quad \forall \mathbf{x} \in \mathcal{O}_{1}, s \in(0,1 / n)
$$

where $L=\sup \left\{|\mathscr{F}(\mathbf{x})|, \mathbf{x} \in \mathcal{O}_{1}\right\}$. Since $f$ is uniformly continuous on $\mathcal{O}_{1}$, it follows that

$$
\lim _{n \rightarrow \infty} \sup \left\{\mid f\left(\mathbf{\Phi}(\mathbf{x},-s)-f(\mathbf{x}) \mid ; \mathbf{x} \in \mathcal{O}_{1}, s \in(0,1 / n)\right\}=0\right.
$$


from which we deduce that there exists some $n_{1} \geqslant 0$, such that $\left|\varrho_{n} \diamond f(\mathbf{x})-f(\mathbf{x})\right| \leqslant \varepsilon$ for any $\mathbf{x} \in \mathcal{O}_{n} \backslash \mathcal{O}_{n}^{-}$and any $n \geqslant n_{1}$. One obtains then, for any $n \geqslant n_{1}$,

$$
\int_{\Omega}\left|\varrho_{n} \diamond f-f\right| \mathrm{d} \mu \leqslant 2 \varepsilon+\varepsilon \mu\left(\mathcal{O}_{n} \backslash \mathcal{O}_{n}^{-}\right) \leqslant 2 \varepsilon+\varepsilon \mu\left(\mathcal{O}_{1}\right)
$$

which proves the result.

We saw that, for a given $f \in L^{1}(\boldsymbol{\Omega}, \mathrm{d} \mu), \varrho_{n} \diamond f$ is also integrable $(n \in \mathbb{N})$. Actually, we shall see that $\varrho_{n} \diamond f$ is even more regular than $f$ :

Lemma 3.9. Given $f \in L^{1}(\boldsymbol{\Omega}, \mathrm{d} \mu)$, set $f_{n}=\varrho_{n} \diamond f, n \in \mathbb{N}$. Then, $f_{n} \in \mathscr{D}\left(\mathcal{T}_{\max }\right)$ with

$$
\left[\mathcal{T}_{\max } f_{n}\right](\mathbf{x})=-\int_{0}^{\tau_{-}(\mathbf{x})} \varrho_{n}^{\prime}(s) f(\mathbf{\Phi}(\mathbf{x},-s)) \mathrm{d} s, \quad \mathbf{x} \in \boldsymbol{\Omega} .
$$

Proof. Set $g_{n}(\mathbf{x})=-\int_{0}^{\tau_{-}(\mathbf{x})} \varrho_{n}^{\prime}(s) f(\boldsymbol{\Phi}(\mathbf{x},-s)) \mathrm{d} s, \mathbf{x} \in \boldsymbol{\Omega}$. It is easy to see that $g_{n} \in L^{1}(\boldsymbol{\Omega}, \mathrm{d} \mu)$. Now, given $\psi \in \mathfrak{Y}$, let us consider the quantity

$$
I=\left.\int_{\Omega} f_{n}(\mathbf{x}) \frac{\mathrm{d}}{\mathrm{d} s} \psi(\mathbf{\Phi}(\mathbf{x}, s))\right|_{s=0} \mathrm{~d} \mu(\mathbf{x}) .
$$

One has to prove that $I=\int_{\boldsymbol{\Omega}} g_{n}(\mathbf{x}) \psi(\mathbf{x}) \mathrm{d} \mu(\mathbf{x})$. We split the above integral over $\boldsymbol{\Omega}$ into three integrals $I_{-}, I_{+}$and $I_{\infty}$ over $\Omega_{-}, \Omega_{-\infty} \cap \Omega_{+}$and $\boldsymbol{\Omega}_{+\infty} \cap \boldsymbol{\Omega}_{-\infty}$ respectively. Recall that, for any $\mathbf{x} \in \boldsymbol{\Omega}_{-}$, there is some $\mathbf{y} \in \Gamma_{-}$and some $t \in\left(0, \tau_{+}(\mathbf{y})\right)$ such that $\mathbf{x}=\mathbf{\Phi}(\mathbf{y}, t)$. In such a case

$$
\left.\frac{\mathrm{d}}{\mathrm{d} s} \psi(\boldsymbol{\Phi}(\mathbf{x}, s))\right|_{s=0}=\frac{\mathrm{d}}{\mathrm{d} t} \psi(\boldsymbol{\Phi}(\mathbf{y}, t)) .
$$

Then, according to Prop. 2.12 and Eq. (3.4):

$$
\begin{aligned}
I_{-} & =\int_{\Gamma_{-}} \mathrm{d} \mu_{-}(\mathbf{y}) \int_{0}^{\tau_{+}(\mathbf{y})} f_{n}(\mathbf{\Phi}(\mathbf{y}, t)) \frac{\mathrm{d}}{\mathrm{d} t} \psi(\mathbf{\Phi}(\mathbf{y}, t)) \mathrm{d} t \\
& =\int_{\Gamma_{-}} \mathrm{d} \mu_{-}(\mathbf{y}) \int_{0}^{\tau_{+}(\mathbf{y})} \frac{\mathrm{d}}{\mathrm{d} t} \psi(\mathbf{\Phi}(\mathbf{y}, t)) \mathrm{d} t \int_{0}^{t} \varrho_{n}(t-s) f(\mathbf{\Phi}(\mathbf{y}, s)) \mathrm{d} s \\
& =\int_{\Gamma_{-}} \mathrm{d} \mu_{-}(\mathbf{y}) \int_{0}^{\tau_{+}(\mathbf{y})} f(\mathbf{\Phi}(\mathbf{y}, s)) \mathrm{d} s \int_{s}^{\tau_{+}(\mathbf{y})} \frac{\mathrm{d}}{\mathrm{d} t} \psi(\mathbf{\Phi}(\mathbf{y}, t)) \varrho_{n}(t-s) \mathrm{d} t .
\end{aligned}
$$

Let us now investigate more carefully this last integral. Let $\mathbf{y} \in \Gamma_{-}$be fixed. If $\tau_{+}(\mathbf{y})<\infty$ then, since $\psi$ is compactly supported, we have $\psi\left(\mathbf{\Phi}\left(\mathbf{y}, \tau_{+}(\mathbf{y})\right)\right)=0$ and integration by part (together with $\varrho_{n}(0)=0$ ) leads to

$$
\int_{s}^{\tau_{+}(\mathbf{y})} \frac{\mathrm{d}}{\mathrm{d} t} \psi(\boldsymbol{\Phi}(\mathbf{y}, t)) \varrho_{n}(t-s) \mathrm{d} t=-\int_{s}^{\tau_{+}(\mathbf{y})} \varrho_{n}^{\prime}(t-s) \psi(\mathbf{\Phi}(\mathbf{y}, t)) \mathrm{d} t .
$$

If now $\tau_{+}(\mathbf{y})>\infty$, then, since $\varrho_{n}$ is supported in $[0,1 / n]$, one has

$$
\begin{aligned}
\int_{s}^{\tau_{+}(\mathbf{y})} \frac{\mathrm{d}}{\mathrm{d} t} \psi(\boldsymbol{\Phi}(\mathbf{y}, t)) \varrho_{n}(t-s) \mathrm{d} t & =\int_{s}^{s+1 / n} \frac{\mathrm{d}}{\mathrm{d} t} \psi(\boldsymbol{\Phi}(\mathbf{y}, t)) \varrho_{n}(t-s) \mathrm{d} t \\
& =-\int_{s}^{\tau_{+}(y)} \varrho_{n}^{\prime}(t-s) \psi(\mathbf{\Phi}(\mathbf{y}, t)) \mathrm{d} t
\end{aligned}
$$


Finally, we obtain,

$$
\begin{aligned}
I_{-} & =-\int_{\Gamma_{-}} \mathrm{d} \mu_{-}(\mathbf{y}) \int_{0}^{\tau_{+}(\mathbf{y})} f(\mathbf{\Phi}(\mathbf{y}, s)) \mathrm{d} s \int_{s}^{\tau_{+}(\mathbf{y})} \psi(\mathbf{\Phi}(\mathbf{y}, t)) \varrho_{n}^{\prime}(t-s) \mathrm{d} t \\
& =-\int_{\Gamma_{-}} \mathrm{d} \mu_{-}(\mathbf{y}) \int_{0}^{\tau_{+}(\mathbf{y})} \psi(\mathbf{\Phi}(\mathbf{y}, t)) \mathrm{d} t \int_{0}^{t} \varrho_{n}^{\prime}(s) f(\mathbf{\Phi}(\mathbf{y}, t-s)) \mathrm{d} s .
\end{aligned}
$$

Using again Prop. 2.12, we finally get

$$
I_{-}=\int_{\Omega_{-}} g_{n}(\mathbf{x}) \psi(\mathbf{x}) \mathrm{d} \mu(\mathbf{x}) .
$$

One proves in the same way that

$$
I_{+}=\left.\int_{\boldsymbol{\Omega}_{+} \cap \boldsymbol{\Omega}_{-\infty}} f_{n}(\mathbf{x}) \frac{\mathrm{d}}{\mathrm{d} s} \psi(\boldsymbol{\Phi}(\mathbf{x}, s))\right|_{s=0} \mathrm{~d} \mu(\mathbf{x})=\int_{\boldsymbol{\Omega}_{+} \cap \boldsymbol{\Omega}_{-\infty}} g_{n}(\mathbf{x}) \psi(\mathbf{x}) \mathrm{d} \mu(\mathbf{x}) .
$$

It remains to consider $I_{\infty}=\left.\int_{\boldsymbol{\Omega}_{+\infty} \cap \boldsymbol{\Omega}_{-\infty}} f_{n}(\mathbf{x}) \frac{\mathrm{d}}{\mathrm{d} s} \psi(\boldsymbol{\Phi}(\mathbf{x}, s))\right|_{s=0} \mathrm{~d} \mu(\mathbf{x})$. One has

$$
\begin{aligned}
I_{\infty} & =\left.\int_{\boldsymbol{\Omega}_{+\infty} \cap \boldsymbol{\Omega}_{-\infty}} \frac{\mathrm{d}}{\mathrm{d} s} \psi(\boldsymbol{\Phi}(\mathbf{x}, s))\right|_{s=0} \mathrm{~d} \mu(\mathbf{x}) \int_{0}^{\infty} \varrho_{n}(t) f(\boldsymbol{\Phi}(\mathbf{x},-t)) \mathrm{d} t \\
& =\left.\int_{0}^{\infty} \varrho_{n}(t) \mathrm{d} t \int_{\boldsymbol{\Omega}_{+\infty} \cap \boldsymbol{\Omega}_{-\infty}} \frac{\mathrm{d}}{\mathrm{d} s} \psi(\boldsymbol{\Phi}(\mathbf{x}, s))\right|_{s=0} f(\boldsymbol{\Phi}(\mathbf{x},-t)) \mathrm{d} \mu(\mathbf{x}) .
\end{aligned}
$$

For any $\mathbf{x} \in \boldsymbol{\Omega}_{+\infty} \cap \boldsymbol{\Omega}_{-\infty}$ and any $t \geqslant 0$, setting $\mathbf{y}=\boldsymbol{\Phi}(\mathbf{x},-t)$, one has $\mathbf{y} \in \boldsymbol{\Omega}_{-\infty} \cap \boldsymbol{\Omega}_{+\infty}$ and $\left.\frac{\mathrm{d}}{\mathrm{d} s} \psi(\boldsymbol{\Phi}(\mathbf{x}, s))\right|_{s=0}=\frac{\mathrm{d}}{\mathrm{d} t} \psi(\boldsymbol{\Phi}(\mathbf{y}, t))$ from which Liouville's Theorem (Assumption 1) yields

$$
\left.\int_{\boldsymbol{\Omega}_{+\infty} \cap \boldsymbol{\Omega}_{-\infty}} \frac{\mathrm{d}}{\mathrm{d} s} \psi(\boldsymbol{\Phi}(\mathbf{x}, s))\right|_{s=0} f(\boldsymbol{\Phi}(\mathbf{x},-t)) \mathrm{d} \mu(\mathbf{x})=\int_{\boldsymbol{\Omega}_{+\infty} \cap \boldsymbol{\Omega}_{-\infty}} \frac{\mathrm{d}}{\mathrm{d} t} \psi(\boldsymbol{\Phi}(\mathbf{y}, t)) f(\mathbf{y}) \mathrm{d} \mu(\mathbf{y}) .
$$

Therefore,

$$
\begin{aligned}
I_{\infty} & =\int_{\boldsymbol{\Omega}_{+\infty} \cap \boldsymbol{\Omega}_{-\infty}} f(\mathbf{y}) \mathrm{d} \mu(\mathbf{y}) \int_{0}^{\infty} \varrho_{n}(t) \frac{\mathrm{d}}{\mathrm{d} t} \psi(\mathbf{\Phi}(\mathbf{y}, t)) \mathrm{d} t \\
& =-\int_{\boldsymbol{\Omega}_{+\infty} \cap \boldsymbol{\Omega}_{-\infty}} f(\mathbf{y}) \mathrm{d} \mu(\mathbf{y}) \int_{0}^{\infty} \varrho_{n}^{\prime}(t) \psi(\mathbf{\Phi}(\mathbf{y}, t)) \mathrm{d} t \\
& =-\int_{0}^{\infty} \varrho_{n}^{\prime}(t) \mathrm{d} t \int_{\boldsymbol{\Omega}_{+\infty} \cap \boldsymbol{\Omega}_{-\infty}} f(\mathbf{y}) \psi(\boldsymbol{\Phi}(\mathbf{y}, t)) \mathrm{d} \mu(\mathbf{y}) .
\end{aligned}
$$

Arguing as above, one can "turn back" to the $\mathrm{x}$ variable to get

$$
\int_{\boldsymbol{\Omega}_{+\infty} \cap \boldsymbol{\Omega}_{-\infty}} f(\mathbf{y}) \psi(\mathbf{\Phi}(\mathbf{y}, t)) \mathrm{d} \mu(\mathbf{y})=\int_{\boldsymbol{\Omega}_{+\infty} \cap \boldsymbol{\Omega}_{-\infty}} f(\mathbf{\Phi}(\mathbf{x},-t)) \psi(\mathbf{x}) \mathrm{d} \mu(\mathbf{x}),
$$

i.e.

$$
I_{\infty}=-\int_{\boldsymbol{\Omega}_{+\infty} \cap \boldsymbol{\Omega}_{-\infty}} \psi(\mathbf{x}) \mathrm{d} \mu(\mathbf{x}) \int_{0}^{\infty} \varrho_{n}^{\prime}(t) f(\mathbf{\Phi}(\mathbf{x},-t)) \mathrm{d} t=\int_{\boldsymbol{\Omega}_{+\infty} \cap \boldsymbol{\Omega}_{-\infty}} \psi(\mathbf{x}) g_{n}(\mathbf{x}) \mathrm{d} \mu(\mathbf{x})
$$

and the Lemma is proven.

Remark 3.10. Notice that Proposition 3.8 together with Lemma 3.9 prove that $\mathscr{D}\left(\mathcal{T}_{\max }\right)$ is a dense subset of $L^{1}(\boldsymbol{\Omega}, \mathrm{d} \mu)$.

Now, whenever $f \in \mathscr{D}\left(\mathcal{T}_{\max }\right)$, one has the following more precise result: 
Proposition 3.11. If $f \in \mathscr{D}\left(\mathcal{T}_{\max }\right)$, then

$$
\left[\mathcal{T}_{\max }\left(\varrho_{n} \diamond f\right)\right](\mathbf{x})=\left[\varrho_{n} \diamond \mathcal{T}_{\max } f\right](\mathbf{x}), \quad(\mathbf{x} \in \mathbf{\Omega}, n \in \mathbb{N}) .
$$

Before proving this result, we need the following very simple lemma:

Lemma 3.12. For any $\psi \in \mathfrak{Y}$ and any $n \in \mathbb{N}$, define

$$
\chi_{n}(\mathbf{x})=\int_{0}^{\tau_{+}(\mathbf{x})} \varrho_{n}(s) \psi(\mathbf{\Phi}(\mathbf{x}, s)) \mathrm{d} s, \quad \mathbf{x} \in \boldsymbol{\Omega} .
$$

Then, $\chi_{n}$ belongs to $\mathfrak{Y}$.

Proof. Since $\tau_{+}$is measurable and $\varrho_{n}$ is compactly supported, it is easy to see that $\chi_{n}$ is measurable and bounded over $\boldsymbol{\Omega}$. Now, for any $\mathbf{x} \in \boldsymbol{\Omega}$, and any $t \in\left(\tau_{-}(\mathbf{x}), \tau_{+}(\mathbf{x})\right)$, one has

$$
\chi_{n}(\mathbf{\Phi}(\mathbf{x}, t))=\int_{t}^{\tau_{+}(\mathbf{x})} \varrho_{n}(s-t) \psi(\mathbf{\Phi}(\mathbf{x}, s)) \mathrm{d} s .
$$

It is clear then from the properties of $\varrho_{n}$ that the mapping $t \in\left(-\tau_{-}(\mathbf{x}), \tau_{+}(\mathbf{x})\right) \mapsto \chi_{n}(\mathbf{\Phi}(\mathbf{x}, t))$ is continuously differentiable with

$$
\frac{\mathrm{d}}{\mathrm{d} t} \chi_{n}(\boldsymbol{\Phi}(\mathbf{x}, t))=-\int_{t}^{\tau_{+}(\mathbf{x})} \varrho_{n}^{\prime}(s-t) \psi(\boldsymbol{\Phi}(\mathbf{x}, s)) \mathrm{d} s=\int_{t}^{\tau_{+}(\mathbf{x})} \varrho_{n}(s-t) \frac{\mathrm{d}}{\mathrm{d} s}[\psi(\boldsymbol{\Phi}(\mathbf{x}, s))] \mathrm{d} s .
$$

In particular, for $t=0$, one gets

$$
\left.\frac{\mathrm{d}}{\mathrm{d} t} \chi_{n}(\mathbf{\Phi}(\mathbf{x}, t))\right|_{t=0}=-\int_{0}^{\tau_{+}(\mathbf{x})} \varrho_{n}^{\prime}(s) \psi(\mathbf{\Phi}(\mathbf{x}, s)) \mathrm{d} s .
$$

Since $\varrho_{n}^{\prime}$ is compactly supported and $\psi \in \mathfrak{Y}$, the application $\left.\mathbf{x} \in \boldsymbol{\Omega} \longmapsto \frac{\mathrm{d}}{\mathrm{d} t} \chi_{n}(\boldsymbol{\Phi}(\mathbf{x}, t))\right|_{t=0}$ is measurable and bounded.

Proof of Proposition 3.11. We use the notations of Lemma 3.9. Since $\varrho_{n} \diamond \mathcal{T}_{\max } f \in$ $L^{1}(\Omega, \mathrm{d} \mu)$, it suffices to show that

$$
\left.\int_{\Omega} f_{n}(\mathbf{x}) \frac{\mathrm{d}}{\mathrm{d} s} \psi(\mathbf{\Phi}(\mathbf{x}, s))\right|_{s=0} \mathrm{~d} \mu(\mathbf{x})=\int_{\Omega} \psi(\mathbf{x})\left[\varrho_{n} \diamond \mathcal{T}_{\max } f\right](\mathbf{x}) \mathrm{d} \mu(\mathbf{x}), \quad \forall \psi \in \mathfrak{Y} .
$$

Here again, we shall deal separately with the integrals over $\boldsymbol{\Omega}_{-}, \boldsymbol{\Omega}_{+} \cap \boldsymbol{\Omega}_{-\infty}$ and $\boldsymbol{\Omega}_{+\infty} \cap \boldsymbol{\Omega}_{-\infty}$. Let $\chi_{n}$ be defined as in Lemma 3.12, as we already saw it (see (3.12)), for any $\mathbf{y} \in \Gamma_{-}$, and any $0<s<\tau_{+}(\mathbf{y}), \frac{\mathrm{d}}{\mathrm{d} s} \chi_{n}(\boldsymbol{\Phi}(\mathbf{y}, s))=\int_{s}^{\tau_{+}(\mathbf{y})} \varrho_{n}(t-s) \frac{\mathrm{d}}{\mathrm{d} t}[\psi(\boldsymbol{\Phi}(\mathbf{y}, t))] \mathrm{d} t$. Consequently, according to (3.10),

$$
\begin{gathered}
\left.\int_{\boldsymbol{\Omega}_{-}} f_{n}(\mathbf{x}) \frac{\mathrm{d}}{\mathrm{d} s} \psi(\mathbf{\Phi}(\mathbf{x}, s))\right|_{s=0} \mathrm{~d} \mu(\mathbf{x})=\int_{\Gamma_{-}} \mathrm{d} \mu(\mathbf{y}) \int_{0}^{\tau_{+}(\mathbf{y})} f(\boldsymbol{\Phi}(\mathbf{y}, r)) \frac{\mathrm{d}}{\mathrm{d} r} \chi_{n}(\mathbf{\Phi}(\mathbf{y}, r)) \mathrm{d} r \\
=\left.\int_{\boldsymbol{\Omega}_{-}} f(\mathbf{x}) \frac{\mathrm{d}}{\mathrm{d} s} \chi_{n}(\mathbf{\Phi}(\mathbf{x}, s))\right|_{s=0} \mathrm{~d} \mu(\mathbf{x})=\int_{\boldsymbol{\Omega}_{-}} \chi_{n}(\mathbf{x})\left[\mathcal{T}_{\max } f\right](\mathbf{x}) \mathrm{d} \mu(\mathbf{x})
\end{gathered}
$$


where, for the two last identities, we used (3.9) and the fact that $\chi_{n} \in \mathfrak{Y}$. Now, using Prop. 2.12

$$
\begin{gathered}
\int_{\boldsymbol{\Omega}_{-}} \chi_{n}(\mathbf{x})\left[\mathcal{T}_{\max } f\right](\mathbf{x}) \mathrm{d} \mu(\mathbf{x})=\int_{\boldsymbol{\Omega}_{-}}\left[\mathcal{T}_{\max } f\right](\mathbf{x}) \mathrm{d} \mu(\mathbf{x}) \int_{0}^{\tau_{+}(\mathbf{x})} \varrho_{n}(r) \psi(\mathbf{\Phi}(\mathbf{x}, r)) \mathrm{d} r \\
=\int_{\Gamma_{-}} \mathrm{d} \mu_{-}(\mathbf{y}) \int_{0}^{\tau_{+}(\mathbf{y})} \psi(\mathbf{\Phi}(\mathbf{y}, s)) \mathrm{d} s \int_{0}^{s} \varrho_{n}(s-t)\left[\mathcal{T}_{\max } f\right](\mathbf{\Phi}(\mathbf{y}, t)) \mathrm{d} t .
\end{gathered}
$$

Therefore, Eq. (3.4) leads to

$$
\begin{aligned}
\int_{\boldsymbol{\Omega}_{-}} \chi_{n}(\mathbf{x})\left[\mathcal{T}_{\max } f\right](\mathbf{x}) \mathrm{d} \mu(\mathbf{x}) & =\int_{\Gamma_{-}} \mathrm{d} \mu_{-}(\mathbf{y}) \int_{0}^{\tau_{+}(\mathbf{y})} \psi(\mathbf{\Phi}(\mathbf{y}, s))\left[\varrho_{n} \diamond \mathcal{T}_{\max } f\right](\mathbf{\Phi}(\mathbf{y}, s)) \mathrm{d} s \\
& =\int_{\boldsymbol{\Omega}_{-}} \psi(\mathbf{x})\left[\varrho_{n} \diamond \mathcal{T}_{\max } f\right](\mathbf{x}) \mathrm{d} \mu(\mathbf{x}) .
\end{aligned}
$$

The integrals over $\boldsymbol{\Omega}_{+} \cap \boldsymbol{\Omega}_{-\infty}$ and $\boldsymbol{\Omega}_{-\infty} \cap \boldsymbol{\Omega}_{+\infty}$ are evaluated in the same way.

We are in position to prove the following

Proposition 3.13. Let $f \in L^{1}(\Omega, \mathrm{d} \mu)$ and $f_{n}=\varrho_{n} \diamond f, n \in \mathbb{N}$. Then, for $\mu_{-}-a$. e. $\mathbf{y} \in \Gamma_{-}$,

$$
f_{n}(\boldsymbol{\Phi}(\mathbf{y}, s))-f_{n}(\mathbf{\Phi}(\mathbf{y}, t))=\int_{s}^{t}\left[\mathcal{T}_{\max } f_{n}\right](\mathbf{\Phi}(\mathbf{y}, r)) \mathrm{d} r \quad \forall 0<s<t<\tau_{+}(\mathbf{y}) .
$$

In the same way, for almost every $\mathbf{z} \in \Gamma_{+}$,

$$
f_{n}(\boldsymbol{\Phi}(\mathbf{z},-s))-f_{n}(\mathbf{\Phi}(\mathbf{z},-t))=\int_{s}^{t} \mathcal{T}_{\max } f_{n}(\mathbf{\Phi}(\mathbf{z},-r)) \mathrm{d} r, \quad \forall 0<s<t<\tau_{-}(\mathbf{z}) .
$$

Proof. We focus only on (3.13), the second assertion following the same lines. Since $f \in$ $L^{1}\left(\boldsymbol{\Omega}_{-}, \mathrm{d} \mu\right)$, Proposition 2.12 implies that the integral $\int_{0}^{\tau_{+}(\mathbf{y})}|f(\boldsymbol{\Phi}(\mathbf{y}, r))| \mathrm{d} r$ exists and is finite for $\mu_{-}$-almost every $\mathbf{y} \in \Gamma_{-}$. Therefore, for $\mu_{-}$-almost every $\mathbf{y} \in \Gamma_{-}$and any $0<t<\tau_{+}(\mathbf{y})$, the quantities $\int_{0}^{t} \varrho_{n}(t-s) f(\boldsymbol{\Phi}(\mathbf{y}, s)) \mathrm{d} s$ and $\int_{0}^{t} \varrho_{n}^{\prime}(t-s) f(\boldsymbol{\Phi}(\mathbf{y}, s)) \mathrm{d} s$ are well-defined and finite. Moreover, thanks to Eq. (3.4) Lemma 3.9, they are respectively equal to $f_{n}(\boldsymbol{\Phi}(\mathbf{y}, t))$ and $\left[\mathcal{T}_{\max } f_{n}\right](\mathbf{\Phi}(\mathbf{y}, t))$. In particular, the mapping $t \in\left(0, \tau_{+}(\mathbf{y})\right) \mapsto\left[\mathcal{T}_{\max } f_{n}\right](\mathbf{\Phi}(\mathbf{y}, t)) \in \mathbb{R}$ is continuous. It is then easy to see that, for any $0<s<t<\tau_{+}(\mathbf{y})$

$$
\begin{aligned}
\int_{s}^{t} & {\left[\mathcal{T}_{\max } f_{n}\right](\mathbf{\Phi}(\mathbf{y}, r)) \mathrm{d} r=-\int_{s}^{t} \mathrm{~d} r \int_{0}^{r} \varrho_{n}^{\prime}(r-u) f(\mathbf{\Phi}(\mathbf{y}, u)) \mathrm{d} u } \\
& =-\int_{0}^{s} f(\mathbf{\Phi}(\mathbf{y}, u)) \mathrm{d} u \int_{s}^{t} \varrho_{n}^{\prime}(r-u) \mathrm{d} r-\int_{s}^{t} f(\mathbf{\Phi}(\mathbf{y}, u)) \mathrm{d} u \int_{u}^{t} \varrho_{n}^{\prime}(r-u) \mathrm{d} r \\
& =-\int_{0}^{t} f(\mathbf{\Phi}(\mathbf{y}, u)) \varrho_{n}(t-u) \mathrm{d} u+\int_{0}^{s} f(\mathbf{\Phi}(\mathbf{y}, u)) \varrho_{n}(s-u) \mathrm{d} u,
\end{aligned}
$$

which is nothing but (3.13).

As a consequence, one gets the following result :

Proposition 3.14. For any $f \in \mathscr{D}\left(\mathcal{T}_{\max }\right)$, there exists some functions $\widetilde{f}_{ \pm} \in L^{1}\left(\boldsymbol{\Omega}_{ \pm}, \mathrm{d} \mu\right)$ such that $\widetilde{f}_{ \pm}(\mathbf{x})=f(\mathbf{x})$ for $\mu$-almost every $\mathbf{x} \in \Omega_{ \pm}$and, for $\mu_{-}$-almost every $\mathbf{y} \in \Gamma_{-}$:

$$
\widetilde{f}_{-}(\mathbf{\Phi}(\mathbf{y}, s))-\widetilde{f}_{-}(\mathbf{\Phi}(\mathbf{y}, t))=\int_{s}^{t}\left[\mathcal{T}_{\max } f\right](\mathbf{\Phi}(\mathbf{y}, r)) \mathrm{d} r \quad \forall 0<s<t<\tau_{+}(\mathbf{y}),
$$


while, for $\mu_{+}$-almost every $\mathbf{z} \in \Gamma_{+}$:

$$
\widetilde{f}_{+}(\boldsymbol{\Phi}(\mathbf{z},-s))-\widetilde{f}_{+}(\boldsymbol{\Phi}(\mathbf{z},-t))=\int_{s}^{t}\left[\mathcal{T}_{\max } f\right](\boldsymbol{\Phi}(\mathbf{z},-r)) \mathrm{d} r \quad \forall 0<s<t<\tau_{-}(\mathbf{z}) .
$$

Proof. Define, for any $n \geqslant 1, f_{n}=\varrho_{n} \diamond f$, so that, from Propositions 3.11 and 3.8, $\lim _{n \rightarrow \infty} \| f_{n}-$ $f\|+\| \mathcal{T}_{\max } f_{n}-\mathcal{T}_{\max } f \|=0$. In particular,

$$
\lim _{n \rightarrow \infty} \int_{\boldsymbol{\Omega}_{-}}\left|f_{n}(\mathbf{x})-f(\mathbf{x})\right|+\left|\left[\mathcal{T}_{\max } f_{n}\right](\mathbf{x})-\left[\mathcal{T}_{\max } f\right](\mathbf{x})\right| \mathrm{d} \mu(\mathbf{x})=0 .
$$

Then Eq. (2.9) yields

$$
\begin{aligned}
\int_{\Gamma_{-}} \mathrm{d} \mu_{-}(\mathbf{y}) \int_{0}^{\tau_{+}(\mathbf{y})} & \left|f_{n}(\mathbf{\Phi}(\mathbf{y}, s))-f(\mathbf{\Phi}(\mathbf{y}, s))\right| \mathrm{d} s \\
& +\int_{\Gamma_{-}} \mathrm{d} \mu_{-}(\mathbf{y}) \int_{0}^{\tau_{+}(\mathbf{y})}\left|\left[\mathcal{T}_{\max } f_{n}\right](\mathbf{\Phi}(\mathbf{y}, s))-\left[\mathcal{T}_{\max } f\right](\mathbf{\Phi}(\mathbf{y}, s))\right| \mathrm{d} s \underset{n \rightarrow \infty}{\longrightarrow} 0
\end{aligned}
$$

since $\mathcal{T}_{\text {max }} f$ and $\mathcal{T}_{\max } f_{n}$ both belong to $L^{1}(\Omega, \mathrm{d} \mu)$. Consequently, for almost every $\mathbf{y} \in \Gamma_{-}$(up to a subsequence, still denoted by $f_{n}$ ) we get

$$
\left\{\begin{array}{l}
f_{n}(\mathbf{\Phi}(\mathbf{y}, \cdot)) \longrightarrow f(\mathbf{\Phi}(\mathbf{y}, \cdot)) \\
\mathcal{T}_{\max } f_{n}(\mathbf{\Phi}(\mathbf{y}, \cdot)) \longrightarrow\left[\mathcal{T}_{\max } f\right](\mathbf{\Phi}(\mathbf{y}, \cdot)) \quad \text { in } \quad L^{1}\left(\left(0, \tau_{+}(\mathbf{y})\right), \mathrm{d} s\right)
\end{array}\right.
$$

as $n \rightarrow \infty$. Let us fix $\mathbf{y} \in \Gamma_{-}$for which this holds. Passing again to a subsequence, we may assume that $f_{n}(\mathbf{\Phi}(\mathbf{y}, s))$ converges (pointwise) to $f(\mathbf{\Phi}(\mathbf{y}, s))$ for almost every $s \in\left(0, \tau_{+}(\mathbf{y})\right)$. Let us fix such a $s_{0}$. Then,

$$
f_{n}\left(\mathbf{\Phi}\left(\mathbf{y}, s_{0}\right)\right)-f_{n}(\mathbf{\Phi}(\mathbf{y}, s))=\int_{s_{0}}^{s}\left[\mathcal{T}_{\max } f_{n}\right](\mathbf{\Phi}(\mathbf{y}, r)) \mathrm{d} r \quad \forall s \in\left(0, \tau_{+}(\mathbf{y})\right) .
$$

Now, the right-hand-side has a limit as $n \rightarrow \infty$ so that the first term on the left-hand side also must converge as $n \rightarrow \infty$. Thus, for any $s \in\left(0, \tau_{+}(\mathbf{y})\right)$, the limit

$$
\lim _{n \rightarrow \infty} f_{n}(\mathbf{\Phi}(\mathbf{y}, s))=\tilde{f}_{-}(\mathbf{\Phi}(\mathbf{y}, s))
$$

exists and, for any $0<s<\tau_{+}(\mathbf{y})$

$$
\widetilde{f}_{-}(\mathbf{\Phi}(\mathbf{y}, s))=\widetilde{f}_{-}\left(\mathbf{\Phi}\left(\mathbf{y}, s_{0}\right)\right)-\int_{s_{0}}^{s}\left[\mathcal{T}_{\max } f\right](\mathbf{\Phi}(\mathbf{y}, r)) \mathrm{d} r .
$$

It is easy to check then that $\widetilde{f}_{-}(\mathbf{x})=f(\mathbf{x})$ for almost every $\mathbf{x} \in \mathbf{\Omega}_{-}$. The same arguments lead to the existence of $\widetilde{f}_{+}$.

The above result shows that the mild formulation of Theorem 3.6 is fulfilled for any $\mathbf{x} \in$ $\boldsymbol{\Omega}_{-} \cup \boldsymbol{\Omega}_{+}$. It remains to deal with $\boldsymbol{\Omega}_{\infty}:=\boldsymbol{\Omega}_{-\infty} \cap \boldsymbol{\Omega}_{+\infty}$.

Proposition 3.15. Let $f \in \mathscr{D}\left(\mathcal{T}_{\max }\right)$. Then, there exists a set $\mathcal{O} \subset \boldsymbol{\Omega}_{\infty}$ with $\mu(\mathcal{O})=0$ and a function $\widetilde{f}$ defined on $\left\{\mathbf{z}=\mathbf{\Phi}(\mathbf{x}, t), \mathbf{x} \in \boldsymbol{\Omega}_{\infty} \backslash \mathcal{O}, t \in \mathbb{R}\right\}$ such that $f(\mathbf{x})=\widetilde{f}(\mathbf{x}) \mu$-almost every $\mathbf{x} \in \boldsymbol{\Omega}_{\infty}$ and

$$
\tilde{f}(\mathbf{\Phi}(\mathbf{x}, s))-\tilde{f}(\mathbf{\Phi}(\mathbf{x}, t))=\int_{s}^{t}\left[\mathcal{T}_{\max } f\right](\mathbf{\Phi}(\mathbf{x}, r)) \mathrm{d} r, \quad \forall \mathbf{x} \in \boldsymbol{\Omega}_{\infty} \backslash \mathcal{O}, s<t .
$$


Proof. Since $(\mathbf{x}, t) \mapsto(\mathbf{z}, t)=(\mathbf{\Phi}(\mathbf{x}, t), t)$ is a measurable and measure preserving mapping from $\boldsymbol{\Omega}_{\infty} \times \mathbb{R}$ onto itself, Propositions 3.8 and 3.11 give

$$
\begin{aligned}
& \lim _{n \rightarrow \infty} \int_{\boldsymbol{\Omega}_{\infty}} \mathrm{d} \mu(\mathbf{x}) \int_{I_{k}}\left|f_{n}(\mathbf{\Phi}(\mathbf{x}, t))-f(\mathbf{\Phi}(\mathbf{x}, t))\right| d t=0 \\
& \lim _{n \rightarrow \infty} \int_{\boldsymbol{\Omega}_{\infty}} \mathrm{d} \mu(\mathbf{x}) \int_{I_{k}}\left|\mathcal{T}_{\max } f_{n}(\mathbf{\Phi}(\mathbf{x}, t))-\mathcal{T}_{\max } f(\mathbf{\Phi}(\mathbf{x}, t))\right| \mathrm{d} t=0,
\end{aligned}
$$

for any $I_{k}=[-k, k], k \in \mathbb{N}$. This shows, in particular, that there is (a maximal) $\mathcal{E} \subset \boldsymbol{\Omega}_{\infty}$ with $\mu(\mathcal{E})=0$ such that, for almost every $\mathbf{x} \in \boldsymbol{\Omega}_{\infty} \backslash \mathcal{E}$ and any bounded interval $I \subset \mathbb{R}$ :

$$
\int_{I}|f(\mathbf{\Phi}(\mathbf{x}, t))| \mathrm{d} t+\int_{I}\left|\left[\mathcal{T}_{\max } f\right](\mathbf{\Phi}(\mathbf{x}, t))\right| \mathrm{d} t<\infty
$$

and we can argue as in Proposition 3.13 that

$$
f_{n}(\mathbf{\Phi}(\mathbf{x}, s))-f_{n}(\mathbf{x})=-\int_{0}^{s} \mathcal{T}_{\max } f_{n}(\mathbf{\Phi}(\mathbf{x}, r)) \mathrm{d} r, \quad \forall s \in \mathbb{R} .
$$

Proposition 3.8 yields the existence of a subsequence $\left(f_{n_{p}}\right)_{p}$ and a $\mu$-null set $A_{0}$ with $\mathcal{E} \subset A_{0} \subset$ $\Omega_{\infty}$ such that

Now, for any $k \in \mathbb{N}$,

$$
\lim _{p \rightarrow \infty} f_{n_{p}}(\mathbf{x})=f(\mathbf{x}), \quad \forall \mathbf{x} \in \boldsymbol{\Omega}_{\infty} \backslash \mathcal{A}_{0}
$$

$$
\lim _{p \rightarrow \infty} \int_{\boldsymbol{\Omega}_{\infty}} \mathrm{d} \mu(\mathbf{x}) \int_{I_{k}}\left|\mathcal{T}_{\max } f_{n_{p}}(\mathbf{\Phi}(\mathbf{x}, t))-\mathcal{T}_{\max } f(\mathbf{\Phi}(\mathbf{x}, t))\right| \mathrm{d} t=0
$$

so that, there is a subsequence (depending on $k$ ) and a $\mu$-null set $A_{k}$ with $A_{0} \subset A_{k} \subset \boldsymbol{\Omega}_{\infty}$ such that

$$
\lim _{p_{(k)} \rightarrow \infty} \int_{I_{k}}\left|\mathcal{T}_{\max } f_{n_{p_{(k)}}}(\mathbf{\Phi}(\mathbf{x}, t))-\mathcal{T}_{\max } f(\mathbf{\Phi}(\mathbf{x}, t))\right| \mathrm{d} t=0, \quad \forall \mathbf{x} \in \boldsymbol{\Omega}_{\infty} \backslash A_{k} .
$$

Let $\mathbf{x} \in \boldsymbol{\Omega}_{\infty} \backslash A_{k}$ and $|s|<k$ be fixed. From

$$
f_{n_{p_{(k)}}}(\mathbf{\Phi}(\mathbf{x}, s))-f_{n_{p_{(k)}}}(\mathbf{x})=-\int_{0}^{s} \mathcal{T}_{\max } f_{n_{p_{(k)}}}(\mathbf{\Phi}(\mathbf{x}, r)) \mathrm{d} r
$$

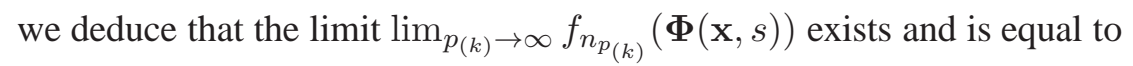

$$
\lim _{p_{(k)} \rightarrow \infty} f_{n_{p_{(k)}}}(\mathbf{\Phi}(\mathbf{x}, s))=f(\mathbf{x})-\int_{0}^{s} \mathcal{T}_{\max } f(\mathbf{\Phi}(\mathbf{x}, r)) \mathrm{d} r .
$$

We define then $\tilde{f}$ by

$$
\widetilde{f}(\mathbf{\Phi}(\mathbf{x}, s))=\lim _{p_{(k)} \rightarrow \infty} f_{n_{p_{(k)}}}(\mathbf{\Phi}(\mathbf{x}, s)), \quad \mathbf{x} \in \boldsymbol{\Omega}_{\infty} \backslash A_{k},|s|<k
$$

and defining $\mathcal{O}=\bigcup_{k \geqslant 1} A_{k}$, we get the result.

Before the proof of Theorem 3.6, we have to establish existence of the trace on $\Gamma_{-}$.

Proposition 3.16. Let $f$ satisfies condition (1) of Theorem 3.6. Then

$$
\lim _{t \rightarrow 0+} f^{\sharp}(\mathbf{\Phi}(\mathbf{y}, t))
$$

exists for almost every $\mathbf{y} \in \Gamma_{-}$. Similarly, $\lim _{t \rightarrow 0+} f^{\sharp}(\mathbf{\Phi}(\mathbf{y},-t))$ exists for almost every $\mathbf{y} \in \Gamma_{+}$. 
Proof. First we note that there is $\widetilde{\Omega}_{-} \subset \Omega_{-}$with $\mu\left(\Omega_{-} \backslash \widetilde{\Omega}_{-}\right)=0$ such that (3.2) is valid any $\mathbf{x} \in \widetilde{\Omega}_{-}$. Let $\widetilde{\Gamma}_{-}=\left\{\mathbf{y} \in \Gamma_{-} ; \mathbf{y}=\mathbf{\Phi}\left(\mathbf{x}, \tau_{-}(\mathbf{x})\right), \mathbf{x} \in \widetilde{\Omega}_{-}\right\}$. It is easy to see that $\mu_{-}\left(\Gamma_{-} \backslash \widetilde{\Gamma}_{-}\right)=0$. Indeed, otherwise, by (2.9), there would be a subset of $\Omega_{-}$of positive $\mu$-measure, not intersecting $\widetilde{\Omega}_{-}$, which would contradict (3.2). Consequently, any $\mathbf{x} \in \widetilde{\Omega}_{-}$can be written as $\mathbf{x}=\Phi\left(\mathbf{y}, \tau_{-}(\mathbf{y})\right)$, $\mathbf{y} \in \widetilde{\Gamma}_{-}$and (3.2) can be recast as

$$
f^{\sharp}(\boldsymbol{\Phi}(\mathbf{y}, t))-f^{\sharp}\left(\boldsymbol{\Phi}\left(\mathbf{y}, t_{0}\right)\right)=\int_{t}^{t_{0}} g(\boldsymbol{\Phi}(\mathbf{y}, s) \mathrm{d} s .
$$

for almost any $\mathbf{y} \in \Gamma_{-}$, where $0<t \leqslant t_{0}<\tau_{+}(\mathbf{y})$. Using again (2.9), $s \mapsto g(\mathbf{\Phi}(\mathbf{y}, s)$ is integrable on $\left(0, \tau_{+}(\mathbf{y})\right.$ for almost any $\mathbf{y} \in \Gamma_{-}$. Consequently, for almost every $\mathbf{y} \in \Gamma_{-}$we can pass to the limit in (3.17) with $t \rightarrow 0$; it is easy to check that this limit does not depend on $t_{0}$. The existence of $\lim _{t \rightarrow 0+} f^{\sharp}(\mathbf{\Phi}(\mathbf{y},-t))$ for a. e. $\mathbf{y} \in \Gamma_{+}$follows by the same argument.

The above proposition allows to define the trace operators.

Definition 3.17. For any $f \in \mathscr{D}\left(\mathcal{T}_{\max }\right)$, define the traces $\mathrm{B}^{ \pm} f$ by

$$
\mathrm{B}^{+} f(\mathbf{y}):=\lim _{t \rightarrow 0+} f^{\sharp}(\mathbf{\Phi}(\mathbf{y},-t)) \quad \text { and } \quad \mathrm{B}^{-} f(\mathbf{y}):=\lim _{t \rightarrow 0+} f^{\sharp}(\mathbf{\Phi}(\mathbf{y}, t))
$$

for any $\mathbf{y} \in \Gamma_{ \pm}$for which the limits exist, where $f^{\sharp}$ is a suitable representative of $f$.

Proof of Theorem 3.6. To prove that $(2) \Longrightarrow(1)$, given $f \in \mathscr{D}\left(\mathcal{T}_{\max }\right)$, set

$$
f^{\natural}(\mathbf{x})= \begin{cases}\tilde{f}_{-}(\mathbf{x}) & \text { if } \mathbf{x} \in \boldsymbol{\Omega}_{-}, \\ \widetilde{f}_{+}(\mathbf{x}) & \text { if } \mathbf{x} \in \boldsymbol{\Omega}_{+} \cap \boldsymbol{\Omega}_{-\infty}, \\ \widetilde{f}(\mathbf{x}) & \text { if } \mathbf{x} \in \boldsymbol{\Omega}_{-\infty} \cap \boldsymbol{\Omega}_{+\infty},\end{cases}
$$

where $\widetilde{f}_{ \pm}$are given by Proposition 3.14 while $\widetilde{f}$ is provided by Prop. 3.15 . Then, it is clear that for any $\mathbf{x} \in \mathbf{\Omega}$ and any $-\tau_{-}(\mathbf{x})<t_{1} \leqslant t_{2}<\tau_{+}(\mathbf{x})$

$$
f^{\sharp}\left(\mathbf{\Phi}\left(\mathbf{x}, t_{1}\right)\right)-f^{\sharp}\left(\mathbf{\Phi}\left(\mathbf{x}, t_{2}\right)\right)=\int_{t_{1}}^{t_{2}}\left[\mathcal{T}_{\max } f\right](\mathbf{\Phi}(\mathbf{x}, s)) \mathrm{d} s
$$

and (3.2) is proven.

Let us now prove that $(1) \Longrightarrow(2)$. Let us fix $\psi \in \mathfrak{Y}$, one has

$$
\begin{aligned}
\left.\int_{\boldsymbol{\Omega}_{-}} f(\mathbf{x}) \frac{\mathrm{d}}{\mathrm{d} s} \psi(\mathbf{\Phi}(\mathbf{x}, s))\right|_{s=0} \mathrm{~d} \mu(\mathbf{x}) & =\int_{\Gamma_{-}} \mathrm{d} \mu_{-}(\mathbf{y}) \int_{0}^{\tau_{+}(\mathbf{y})} f(\boldsymbol{\Phi}(\mathbf{y}, t)) \frac{\mathrm{d}}{\mathrm{d} t} \psi(\mathbf{\Phi}(\mathbf{y}, t)) \mathrm{d} t \\
& =\int_{\Gamma_{-}} \mathrm{d} \mu_{-}(\mathbf{y}) \int_{0}^{\tau_{+}(\mathbf{y})} f^{\sharp}(\mathbf{\Phi}(\mathbf{y}, t)) \frac{\mathrm{d}}{\mathrm{d} t} \psi(\boldsymbol{\Phi}(\mathbf{y}, t)) \mathrm{d} t .
\end{aligned}
$$

Notice that since both $\left.\int_{\boldsymbol{\Omega}_{-}} f(\mathbf{x}) \frac{\mathrm{d}}{\mathrm{d} s} \psi(\boldsymbol{\Phi}(\mathbf{x}, s))\right|_{s=0} \mathrm{~d} \mu(\mathbf{x})$ and $\int_{\boldsymbol{\Omega}_{-}} \psi(\mathbf{x}) g(\mathbf{x}) \mathrm{d} \mu(\mathbf{x})$ exist, Proposition 2.12 and Fubini's Theorem, the integrals

$$
\int_{0}^{\tau_{+}(\mathbf{y})} f^{\sharp}(\mathbf{\Phi}(\mathbf{y}, t)) \frac{\mathrm{d}}{\mathrm{d} t} \psi(\boldsymbol{\Phi}(\mathbf{y}, t)) \mathrm{d} t \quad \text { and } \quad \int_{0}^{\tau_{+}(\mathbf{y})} g(\boldsymbol{\Phi}(\mathbf{y}, t)) \psi(\boldsymbol{\Phi}(\mathbf{y}, t)) \mathrm{d} t
$$

are well-defined for $\mu_{-}$-almost every $\mathbf{y} \in \Gamma_{-}$. Let us prove that these two integrals coincide for almost-every $\mathbf{y} \in \Gamma_{-}$. According to Lemma 3.3, for almost every $\mathbf{y} \in \Gamma_{-}$, there is a sequence 
$\left(t_{n}\right)_{n}$ (depending on $\mathbf{y}$ ) such that $\psi\left(\mathbf{\Phi}\left(\mathbf{y}, t_{n}\right)\right)=0$ and $t_{n} \rightarrow \tau_{+}(\mathbf{y})$. Thus,

$$
\int_{0}^{\tau_{+}(\mathbf{y})} f^{\sharp}(\mathbf{\Phi}(\mathbf{y}, t)) \frac{\mathrm{d}}{\mathrm{d} t} \psi(\boldsymbol{\Phi}(\mathbf{y}, t)) \mathrm{d} t=\lim _{n \rightarrow \infty} \int_{0}^{t_{n}} f^{\sharp}(\boldsymbol{\Phi}(\mathbf{y}, t)) \frac{\mathrm{d}}{\mathrm{d} t} \psi(\boldsymbol{\Phi}(\mathbf{y}, t)) \mathrm{d} t
$$

and

$$
\int_{0}^{\tau_{+}(\mathbf{y})} g(\mathbf{\Phi}(\mathbf{y}, t)) \psi(\mathbf{\Phi}(\mathbf{y}, t)) \mathrm{d} t=\lim _{n \rightarrow \infty} \int_{0}^{t_{n}} \psi(\mathbf{\Phi}(\mathbf{y}, t)) g(\mathbf{\Phi}(\mathbf{y}, t)) \mathrm{d} t .
$$

Further, for almost every $\mathbf{y} \in \Gamma_{-}$, according to (3.2),

$$
f^{\sharp}(\boldsymbol{\Phi}(\mathbf{y}, t))=\mathrm{B}^{-} f(\mathbf{y})-\int_{0}^{t} g(\boldsymbol{\Phi}(\mathbf{y}, r)) \mathrm{d} r, \quad \forall t \in\left(0, \tau_{+}(\mathbf{y})\right) .
$$

Integration by parts, using the fact that $\psi(\mathbf{\Phi}(\mathbf{y}, 0))=\psi\left(\mathbf{\Phi}\left(\mathbf{y}, t_{n}\right)\right)=0$ for any $n$, leads to

$$
\int_{0}^{t_{n}} f^{\sharp}(\boldsymbol{\Phi}(\mathbf{y}, t)) \frac{\mathrm{d}}{\mathrm{d} t} \psi(\boldsymbol{\Phi}(\mathbf{y}, t)) \mathrm{d} t=\int_{0}^{t_{n}} g(\boldsymbol{\Phi}(\mathbf{y}, t)) \psi(\boldsymbol{\Phi}(\mathbf{y}, t)) \mathrm{d} t .
$$

Consequently, for $\mu_{-}$almost every $\mathbf{y} \in \Gamma_{-}$:

$$
\int_{0}^{\tau_{+}(\mathbf{y})} f^{\sharp}(\mathbf{\Phi}(\mathbf{y}, t)) \frac{\mathrm{d}}{\mathrm{d} t} \psi(\mathbf{\Phi}(\mathbf{y}, t)) \mathrm{d} t=\int_{0}^{\tau_{+}(\mathbf{y})} \psi(\mathbf{\Phi}(\mathbf{y}, s)) g(\mathbf{\Phi}(\mathbf{y}, t)) \mathrm{d} t .
$$

Finally, we get

$$
\begin{aligned}
\left.\int_{\boldsymbol{\Omega}_{-}} f(\mathbf{x}) \frac{\mathrm{d}}{\mathrm{d} s} \psi(\boldsymbol{\Phi}(\mathbf{x}, s))\right|_{s=0} \mathrm{~d} \mu(\mathbf{x}) & =\int_{\Gamma_{-}} \mathrm{d} \mu_{-}(\mathbf{y}) \int_{0}^{\tau_{+}(\mathbf{y})} \psi(\mathbf{\Phi}(\mathbf{y}, t)) g(\mathbf{\Phi}(\mathbf{y}, t)) \mathrm{d} t \\
& =\int_{\boldsymbol{\Omega}_{-}} g(\mathbf{x}) \psi(\mathbf{x}) \mathrm{d} \mu(\mathbf{x}) .
\end{aligned}
$$

Using now parametrization over $\Gamma_{+}$, we prove in the same way that

$$
\left.\int_{\boldsymbol{\Omega}_{+} \cap \boldsymbol{\Omega}_{-\infty}} f(\mathbf{x}) \frac{\mathrm{d}}{\mathrm{d} s} \psi(\mathbf{\Phi}(\mathbf{x}, s))\right|_{s=0} \mathrm{~d} \mu(\mathbf{x})=\int_{\boldsymbol{\Omega}_{+} \cap \boldsymbol{\Omega}_{-\infty}} g(\mathbf{x}) \psi(\mathbf{x}) \mathrm{d} \mu(\mathbf{x}) .
$$

It remains now to evaluate $A:=\left.\int_{\boldsymbol{\Omega}_{+\infty} \cap \boldsymbol{\Omega}_{-\infty}} f(\mathbf{x}) \frac{\mathrm{d}}{\mathrm{d} s} \psi(\boldsymbol{\Phi}(\mathbf{x}, s))\right|_{s=0} \mathrm{~d} \mu(\mathbf{x})$. According to Assumption 1

$$
A=\int_{\boldsymbol{\Omega}_{+\infty} \cap \boldsymbol{\Omega}_{-\infty}} f^{\sharp}(\boldsymbol{\Phi}(\mathbf{x}, t)) \frac{\mathrm{d}}{\mathrm{d} t} \psi(\boldsymbol{\Phi}(\mathbf{x}, t)) \mathrm{d} \mu(\mathbf{x}), \quad \forall t \in \mathbb{R} .
$$

Let us integrate the above identity over $(0,1)$, so that

$$
A=\int_{\boldsymbol{\Omega}_{-\infty} \cap \boldsymbol{\Omega}_{+\infty}} \mathrm{d} \mu(\mathbf{x}) \int_{0}^{1} f^{\sharp}(\boldsymbol{\Phi}(\mathbf{x}, t)) \frac{\mathrm{d}}{\mathrm{d} t} \psi(\boldsymbol{\Phi}(\mathbf{x}, t)) \mathrm{d} t .
$$

Let us fix x $\in \boldsymbol{\Omega}_{-\infty} \cap \boldsymbol{\Omega}_{+\infty}$. For any $t \in(0,1)$, one has $f^{\sharp}(\boldsymbol{\Phi}(\mathbf{x}, t))=f^{\sharp}(\mathbf{x})-\int_{0}^{t} g(\boldsymbol{\Phi}(\mathbf{x}, s)) \mathrm{d} s$ and integration by parts yields

$$
\begin{aligned}
\int_{0}^{1} f^{\sharp}(\boldsymbol{\Phi}(\mathbf{x}, t)) \frac{\mathrm{d}}{\mathrm{d} t} \psi(\mathbf{\Phi}(\mathbf{x}, t)) \mathrm{d} t=\int_{0}^{1} \psi(\mathbf{\Phi}(\mathbf{x}, t)) g(\mathbf{\Phi}(\mathbf{x}, t)) \mathrm{d} t-\psi(\mathbf{x}) f^{\sharp}(\mathbf{x}) \\
+\psi(\mathbf{\Phi}(\mathbf{x}, 1))\left(f^{\sharp}(\mathbf{x})-\int_{0}^{1} g(\mathbf{\Phi}(\mathbf{x}, s)) \mathrm{d} s\right) \\
=\int_{0}^{1} \psi(\mathbf{\Phi}(\mathbf{x}, t)) g(\mathbf{\Phi}(\mathbf{x}, t)) \mathrm{d} t+\psi(\mathbf{\Phi}(\mathbf{x}, 1)) f^{\sharp}(\mathbf{\Phi}(\mathbf{x}, 1))-\psi(\mathbf{x}) f^{\sharp}(\mathbf{x})
\end{aligned}
$$


where we used again (3.2). Integrating over $\boldsymbol{\Omega}_{-\infty} \cap \boldsymbol{\Omega}_{+\infty}$ we see from Liouville's Theorem (Assumption 1) that

$$
\int_{\boldsymbol{\Omega}_{-\infty} \cap \boldsymbol{\Omega}_{+\infty}} \psi(\boldsymbol{\Phi}(\mathbf{x}, 1)) f^{\sharp}(\mathbf{\Phi}(\mathbf{x}, 1)) \mathrm{d} \mu(\mathbf{x})=\int_{\boldsymbol{\Omega}_{-\infty} \cap \boldsymbol{\Omega}_{+\infty}} \psi(\mathbf{x}) f^{\sharp}(\mathbf{x}) \mathrm{d} \mu(\mathbf{x}),
$$

i.e.

$$
A=\int_{\boldsymbol{\Omega}_{-\infty} \cap \boldsymbol{\Omega}_{+\infty}} \mathrm{d} \mu(\mathbf{x}) \int_{0}^{1} \psi(\boldsymbol{\Phi}(\mathbf{x}, t)) g(\mathbf{\Phi}(\mathbf{x}, t)) \mathrm{d} t
$$

which, thanks to Liouville's Theorem, is nothing but

$$
\left.\int_{\boldsymbol{\Omega}_{+\infty} \cap \boldsymbol{\Omega}_{-\infty}} f(\mathbf{x}) \frac{\mathrm{d}}{\mathrm{d} s} \psi(\mathbf{\Phi}(\mathbf{x}, s))\right|_{s=0} \mathrm{~d} \mu(\mathbf{x})=\int_{\boldsymbol{\Omega}_{-\infty} \cap \boldsymbol{\Omega}_{+\infty}} g(\mathbf{x}) \psi(\mathbf{x}) \mathrm{d} \mu(\mathbf{x}) .
$$

Combining (3.19), (3.20) and (3.21), we obtain

$$
\left.\int_{\boldsymbol{\Omega}} f(\mathbf{x}) \frac{\mathrm{d}}{\mathrm{d} s} \psi(\mathbf{\Phi}(\mathbf{x}, s))\right|_{s=0} \mathrm{~d} \mu(\mathbf{x})=\int_{\boldsymbol{\Omega}} g(\mathbf{x}) \psi(\mathbf{x}) \mathrm{d} \mu(\mathbf{x}), \quad \forall \psi \in \mathfrak{Y}
$$

which exactly means that $f \in \mathscr{D}\left(\mathcal{T}_{\max }\right)$ with $g=\mathcal{T}_{\text {max }}$ and the proof is complete.

Corollary 3.18. Traces $B^{ \pm} f$ on $\Gamma_{ \pm}$can be defined for any $f \in \mathscr{D}\left(\mathcal{T}_{\max }\right)$. For $\mu_{-}$- almost any $\mathbf{y} \in \Gamma_{-}$we have

$$
\mathrm{B}^{-} f(\mathbf{y})=f^{\sharp}(\mathbf{\Phi}(\mathbf{y}, t))+\int_{0}^{t}\left[\mathcal{T}_{\max } f\right](\mathbf{\Phi}(y, s)) \mathrm{d} s, \quad \forall t \in\left(0, \tau_{+}(y)\right),
$$

where $f^{\sharp}$ is a suitable representative of $f$. An analogous formula holds for $\mathrm{B}^{+} f$.

Lemma 2.9 provides the existence of Borel measures $\mathrm{d} \mu_{ \pm}$on $\Gamma_{ \pm}$, which allow us to define the natural trace spaces associated to Problem (1.1), namely,

$$
L_{ \pm}^{1}:=L^{1}\left(\Gamma_{ \pm}, \mathrm{d} \mu_{ \pm}\right)
$$

However, the traces $B^{ \pm} f, f \in \mathscr{D}\left(\mathcal{T}_{\max }\right)$, not necessarily belong to $L_{ \pm}^{1}$.

\section{WELL-POSEDNESS FOR INITIAL AND BOUNDARY- VALUE PROBLEMS}

4.1. Absorption semigroup. From now on, we will denote $X=L^{1}(\boldsymbol{\Omega}, \mathrm{d} \mu)$ endowed with its natural norm $\|\cdot\|_{X}$. Let $\mathcal{T}_{0}$ be the free streaming operator with no re-entry boundary conditions:

$$
\mathcal{T}_{0} \psi=\mathcal{T}_{\max } \psi, \quad \text { for any } \psi \in \mathscr{D}\left(\mathcal{T}_{0}\right),
$$

where the domain $\mathscr{D}\left(\mathcal{T}_{0}\right)$ is defined by

$$
\mathscr{D}\left(\mathcal{T}_{0}\right)=\left\{\psi \in \mathscr{D}\left(\mathcal{T}_{\text {max }}\right) ; \mathrm{B}^{-} \psi=0\right\}
$$

We state the following generation result:

Theorem 4.1. The operator $\left(\mathcal{T}_{0}, \mathscr{D}\left(\mathcal{T}_{0}\right)\right)$ is the generator of a nonnegative $C_{0}$-semigroup of contractions $\left(U_{0}(t)\right)_{t \geqslant 0}$ in $L^{1}(\Omega, \mathrm{d} \mu)$ given by

$$
U_{0}(t) f(\mathbf{x})=f(\mathbf{\Phi}(\mathbf{x},-t)) \chi_{\left\{t<\tau_{-}(\mathbf{x})\right\}}(\mathbf{x}), \quad(\mathbf{x} \in \mathbf{\Omega}, f \in X),
$$

where $\chi_{A}$ denotes the characteristic function of a set $A$. 
Proof. The proof is divided into three steps:

- Step 1. Let us first check that the family of operators $\left(U_{0}(t)\right)_{t \geqslant 0}$ is a nonnegative contractive $C_{0}$-semigroup in $X$. Thanks to Proposition 2.3, we can prove that, for any $f \in X$ and any $t \geqslant 0$, the mapping $U_{0}(t) f: \Omega \rightarrow \mathbb{R}$ is measurable and the semigroup properties $U_{0}(0) f=f$ and $U_{0}(t) U_{0}(s) f=U_{0}(t+s) f(t, s \geqslant 0)$ hold. Let us now show that $\left\|U_{0}(t) f\right\|_{X} \leqslant\|f\|_{X}$. We have

$$
\left\|U_{0}(t) f\right\|_{X}=\int_{\boldsymbol{\Omega}_{+}}\left|U_{0}(t) f\right| \mathrm{d} \mu+\int_{\boldsymbol{\Omega}_{-} \cap \boldsymbol{\Omega}_{+\infty}}\left|U_{0}(t) f\right| \mathrm{d} \mu+\int_{\boldsymbol{\Omega}_{-\infty} \cap \boldsymbol{\Omega}_{+\infty}}\left|U_{0}(t) f\right| \mathrm{d} \mu .
$$

Propositions 2.12 and 2.3 yield

$$
\begin{aligned}
\int_{\boldsymbol{\Omega}_{+}}\left|U_{0}(t) f\right| \mathrm{d} \mu & =\int_{\Gamma_{+}} \mathrm{d} \mu_{+}(\mathbf{y}) \int_{0}^{\tau_{-}(\mathbf{y})}\left|U_{0}(t) f(\mathbf{\Phi}(\mathbf{y},-s))\right| \mathrm{d} s \\
& =\int_{\Gamma_{+}} \mathrm{d} \mu_{+}(\mathbf{y}) \int_{0}^{\max \left(0, \tau_{-}(\mathbf{y})-t\right)}|f(\mathbf{\Phi}(\mathbf{y},-s-t))| \mathrm{d} s \\
& \leqslant \int_{\Gamma_{+}} \mathrm{d} \mu_{+}(\mathbf{y}) \int_{t}^{\max \left(t, \tau_{-}(\mathbf{y})\right)}|f(\mathbf{\Phi}(\mathbf{y},-r))| \mathrm{d} r \leqslant \int_{\Omega_{+}}|f| \mathrm{d} \mu .
\end{aligned}
$$

In the same way we obtain

$$
\int_{\boldsymbol{\Omega}_{-} \cap \boldsymbol{\Omega}_{+\infty}}\left|U_{0}(t) f\right| \mathrm{d} \mu=\int_{\Gamma_{-\infty}} \mathrm{d} \mu_{-}(\mathbf{y}) \int_{0}^{\infty}\left|U_{0}(t) f(\mathbf{\Phi}(\mathbf{y}, s))\right| \mathrm{d} s=\int_{\boldsymbol{\Omega}_{-} \cap \boldsymbol{\Omega}_{+\infty}}|f| \mathrm{d} \mu,
$$

and

$$
\int_{\boldsymbol{\Omega}_{-\infty} \cap \boldsymbol{\Omega}_{+\infty}}\left|U_{0}(t) f\right| \mathrm{d} \mu=\int_{\boldsymbol{\Omega}_{-\infty} \cap \boldsymbol{\Omega}_{+\infty}}|f| \mathrm{d} \mu .
$$

This proves contractivity of $U_{0}(t)$. Let us now show that $U_{0}(t) f$ is continuous, i.e.

$$
\lim _{t \rightarrow 0}\left\|U_{0}(t) f-f\right\|_{X}=0 .
$$

It is enough to show that this property holds for any $f \in \mathscr{C}_{0}(\boldsymbol{\Omega})$. In this case, $\lim _{t \rightarrow 0} U_{0}(t) f(\mathbf{x})=$ $f(\mathbf{x})$ for any $\mathbf{x} \in \boldsymbol{\Omega}$. Moreover, $\sup _{\mathbf{x} \in \boldsymbol{\Omega}}\left|U_{0}(t) f(\mathbf{x})\right| \leqslant \sup _{\mathbf{x} \in \boldsymbol{\Omega}}|f(\mathbf{x})|$ and the support of $U_{0}(t) f$ is bounded, so that the Lebesgue dominated convergence theorem leads to the result. This proves that $\left(U_{0}(t)\right)_{t \geqslant 0}$ is a $C_{0}$-semigroup of contractions in $X$. Let $\mathcal{A}_{0}$ denote its generator.

- Step 2. To show that $\mathscr{D}\left(\mathcal{A}_{0}\right) \subset \mathscr{D}\left(\mathcal{T}_{0}\right)$, fix $f \in \mathscr{D}\left(\mathcal{A}_{0}\right), \lambda>0$ and $g=\left(\lambda-\mathcal{A}_{0}\right) f$. Then,

$$
f(\mathbf{x})=\int_{0}^{\tau_{-}(\mathbf{x})} \exp (-\lambda t) g(\mathbf{\Phi}(\mathbf{x},-t)) \mathrm{d} t, \quad(\mathbf{x} \in \mathbf{\Omega}) .
$$

To prove that $f \in \mathscr{D}\left(\mathcal{T}_{\text {max }}\right)$ with $\mathcal{T}_{\text {max }} f=\mathcal{A}_{0} f$, it suffices to prove that

$$
\int_{\boldsymbol{\Omega}}(\lambda f(\mathbf{x})-g(\mathbf{x})) \psi(\mathbf{x}) \mathrm{d} \mu(\mathbf{x})=\left.\int_{\boldsymbol{\Omega}} f(\mathbf{x}) \frac{\mathrm{d}}{\mathrm{d} s} \psi(\mathbf{\Phi}(\mathbf{x}, s))\right|_{s=0} \mathrm{~d} \mu(\mathbf{x}), \quad \forall \psi \in \mathfrak{Y} .
$$

Let us fix $\psi \in \mathfrak{Y}$, set $\varphi(\mathbf{x}):=\left.\frac{\mathrm{d}}{\mathrm{d} s} \psi(\boldsymbol{\Phi}(\mathbf{x}, s))\right|_{s=0}$ and write

$$
\begin{aligned}
\int_{\boldsymbol{\Omega}} f(\mathbf{x}) \varphi(\mathbf{x}) \mathrm{d} \mu(\mathbf{x})=\int_{\boldsymbol{\Omega}_{+}} f(\mathbf{x}) \varphi(\mathbf{x}) \mathrm{d} \mu(\mathbf{x}) & +\int_{\boldsymbol{\Omega}_{+\infty} \cap \boldsymbol{\Omega}_{-}} f(\mathbf{x}) \varphi(\mathbf{x}) \mathrm{d} \mu(\mathbf{x}) \\
& +\int_{\boldsymbol{\Omega}_{+\infty} \cap \boldsymbol{\Omega}_{-\infty}} f(\mathbf{x}) \varphi(\mathbf{x}) \mathrm{d} \mu(\mathbf{x})=I_{1}+I_{2}+I_{3} .
\end{aligned}
$$


We first deal with $I_{1}$. For any $\mathbf{y} \in \Gamma_{+}$and $t \in\left(0, \tau_{-}(\mathbf{y})\right)$ we have $\varphi(\boldsymbol{\Phi}(\mathbf{y},-t))=-\frac{\mathrm{d}}{\mathrm{d} t} \psi(\boldsymbol{\Phi}(\mathbf{y},-t))$ and $f(\boldsymbol{\Phi}(\mathbf{y},-t))=\int_{t}^{\tau_{-}(\mathbf{y})} \exp (-\lambda(s-t)) g(\mathbf{\Phi}(\mathbf{y},-s)) \mathrm{d} s$. Then, by Proposition 2.12,

$$
\begin{aligned}
I_{1}= & -\int_{\Gamma_{+}} \mathrm{d} \mu_{+}(\mathbf{y}) \int_{0}^{\tau_{-}(\mathbf{y})} \frac{\mathrm{d}}{\mathrm{d} t} \psi(\boldsymbol{\Phi}(\mathbf{y},-t)) \mathrm{d} t \int_{t}^{\tau_{-}(\mathbf{y})} \exp (-\lambda(s-t)) g(\mathbf{\Phi}(\mathbf{y},-s)) \mathrm{d} s \\
= & -\int_{\Gamma_{+}} \mathrm{d} \mu_{+}(\mathbf{y}) \int_{0}^{\tau_{-}(\mathbf{y})} g(\mathbf{\Phi}(\mathbf{y},-s)) \mathrm{d} s \int_{0}^{s} \exp (-\lambda(s-t)) \frac{\mathrm{d}}{\mathrm{d} t}(\psi(\mathbf{\Phi}(\mathbf{y},-t))) \mathrm{d} t \\
= & \int_{\Gamma_{+}} \mathrm{d} \mu_{+}(\mathbf{y}) \int_{0}^{\tau_{-}(\mathbf{y})} g(\mathbf{\Phi}(\mathbf{y},-s)) \times \\
& \times\left\{\lambda \int_{0}^{s} \exp (-\lambda(s-t)) \psi(\mathbf{\Phi}(\mathbf{y},-t)) \mathrm{d} t-\psi(\mathbf{\Phi}(\mathbf{y},-s))\right\} \mathrm{d} s
\end{aligned}
$$

where we used the fact that $\psi(\mathbf{\Phi}(\mathbf{y}, 0))=0$ for any $\mathbf{y} \in \Gamma_{+}$since $\psi$ is compactly supported. Thus

$$
\begin{array}{r}
I_{1}=\lambda \int_{\Gamma_{+}} \mathrm{d} \mu_{+}(\mathbf{y}) \int_{0}^{\tau_{-}(\mathbf{y})} \psi(\mathbf{\Phi}(\mathbf{y},-t)) \mathrm{d} t \int_{t}^{\tau_{-}(\mathbf{y})} \exp (-\lambda(s-t)) g(\mathbf{\Phi}(\mathbf{y},-s)) \mathrm{d} s \\
-\int_{\Gamma_{-}} \mathrm{d} \mu_{+}(\mathbf{y}) \int_{0}^{\tau_{-}(\mathbf{y})} g(\mathbf{\Phi}(\mathbf{y},-s)) \psi(\mathbf{\Phi}(\mathbf{y},-s)) \mathrm{d} s \\
=\int_{\Gamma_{+}} \mathrm{d} \mu_{+}(\mathbf{y}) \int_{0}^{\tau_{-}(\mathbf{y})} \psi(\mathbf{\Phi}(\mathbf{y},-t))(\lambda f(\mathbf{\Phi}(\mathbf{y},-t))-g(\mathbf{\Phi}(\mathbf{y},-t))) \mathrm{d} t .
\end{array}
$$

Using again Proposition 2.12, we obtain

$$
I_{1}=\int_{\boldsymbol{\Omega}_{+}}(\lambda f(\mathbf{x})-g(\mathbf{x})) \psi(\mathbf{x}) \mathrm{d} \mu(\mathbf{x}) .
$$

Arguing in a similar way, we prove that

$$
I_{2}=-\int_{\boldsymbol{\Omega}_{-} \cap \boldsymbol{\Omega}_{+\infty}}(\lambda f(\mathbf{x})-g(\mathbf{x})) \psi(\mathbf{x}) \mathrm{d} \mu(\mathbf{x}) .
$$

Finally, since

$$
f(\mathbf{x})=\int_{0}^{\infty} \exp (-\lambda t) g(\boldsymbol{\Phi}(\mathbf{x},-t)) \mathrm{d} t \quad \text { for any } \quad \mathbf{x} \in \boldsymbol{\Omega}_{-\infty} \cap \boldsymbol{\Omega}_{+\infty},
$$

one has

$$
\begin{aligned}
I_{3} & =\int_{\boldsymbol{\Omega}_{-\infty} \cap \boldsymbol{\Omega}_{+\infty}} \varphi(\mathbf{x}) \mathrm{d} \mu(\mathbf{x}) \int_{0}^{\infty} \exp (-\lambda t) g(\mathbf{\Phi}(\mathbf{x},-t)) \mathrm{d} t \\
& =\int_{0}^{\infty} \exp (-\lambda t) \mathrm{d} t \int_{\boldsymbol{\Omega}_{-\infty} \cap \boldsymbol{\Omega}_{+\infty}} \varphi(\mathbf{x}) g(\mathbf{\Phi}(\mathbf{x},-t)) \mathrm{d} \mu(\mathbf{x}) .
\end{aligned}
$$

Now, Assumption 1 asserts that

$$
\int_{\boldsymbol{\Omega}_{-\infty} \cap \boldsymbol{\Omega}_{+\infty}} \varphi(\mathbf{x}) g(\boldsymbol{\Phi}(\mathbf{x},-t)) \mathrm{d} \mu(\mathbf{x})=\int_{\boldsymbol{\Omega}_{-\infty} \cap \boldsymbol{\Omega}_{+\infty}} g(\mathbf{x}) \varphi(\mathbf{\Phi}(\mathbf{x}, t)) \mathrm{d} \mu(\mathbf{x}), \quad \forall t \geqslant 0,
$$


and, since $\varphi(\boldsymbol{\Phi}(\mathbf{x}, t))=\frac{\mathrm{d}}{\mathrm{d} t} \psi(\boldsymbol{\Phi}(\mathbf{x}, t))$, finally

$$
\begin{aligned}
I_{3} & =\int_{\boldsymbol{\Omega}_{-\infty} \cap \boldsymbol{\Omega}_{+\infty}} g(\mathbf{x}) \mathrm{d} \mu(\mathbf{x}) \int_{0}^{\infty} \exp (-\lambda t) \frac{\mathrm{d}}{\mathrm{d} t}(\psi(\mathbf{\Phi}(\mathbf{x}, t))) \mathrm{d} t \\
& =-\int_{\boldsymbol{\Omega}_{-\infty} \cap \boldsymbol{\Omega}_{+\infty}} g(\mathbf{x}) \psi(\mathbf{x}) \mathrm{d} \mu(\mathbf{x})+\lambda \int_{\boldsymbol{\Omega}_{-\infty} \cap \boldsymbol{\Omega}_{+\infty}} g(\mathbf{x}) \mathrm{d} \mu(\mathbf{x}) \int_{0}^{\infty} \exp (-\lambda t) \psi(\boldsymbol{\Phi}(\mathbf{x}, t)) \mathrm{d} t .
\end{aligned}
$$

Using again Assumption 1, this finally gives

$$
I_{3}=-\int_{\boldsymbol{\Omega}_{-\infty} \cap \boldsymbol{\Omega}_{+\infty}}(g(\mathbf{x})-\lambda f(\mathbf{x})) \psi(\mathbf{x}) \mathrm{d} \mu(\mathbf{x}) .
$$

Combining (4.2)-(4.4) leads to

$$
\left.\int_{\boldsymbol{\Omega}} f(\mathbf{x}) \frac{\mathrm{d}}{\mathrm{d} s} \psi(\mathbf{\Phi}(\mathbf{x}, s))\right|_{s=0} \mathrm{~d} \mu(\mathbf{x})=-\int_{\boldsymbol{\Omega}}(g(\mathbf{x})-\lambda f(\mathbf{x})) \psi(\mathbf{x}) \mathrm{d} \mu(\mathbf{x})
$$

which proves that $f \in \mathscr{D}\left(\mathcal{T}_{\max }\right)$ and $\left(\lambda-\mathcal{T}_{\max }\right) f=g$. Next, for $\mathbf{y} \in \Gamma_{-}$and $0<t<\tau_{+}(\mathbf{y})$ we write $t=\tau_{-}(\boldsymbol{\Phi}(\mathbf{y}, t))$ and, by Proposition 2.3 and (4.1), we obtain

$$
f(\boldsymbol{\Phi}(\mathbf{y}, t))=\int_{0}^{t} \exp (-\lambda(t-s)) g(\boldsymbol{\Phi}(\mathbf{y}, s)) \mathrm{d} s .
$$

Consequently, $\lim _{t \rightarrow 0^{+}} f(\boldsymbol{\Phi}(\mathbf{y}, t))=0$ a.e. $\mathbf{y} \in \Gamma_{-}$, i.e. $\mathbf{B}^{-} f=0$ so that $f \in \mathscr{D}\left(\mathcal{T}_{0}\right)$ and $\mathcal{A}_{0} f=\mathcal{T}_{0} f=\lambda f-g$.

- Step 3. Now let us show the converse inclusion $\mathscr{D}\left(\mathcal{T}_{0}\right) \subset \mathscr{D}\left(\mathcal{A}_{0}\right)$. Let $f \in \mathscr{D}\left(\mathcal{T}_{0}\right)$. Changing possibly $f$ on a set of zero measure, we may write $f=f^{\sharp}$, where $f^{\sharp}$ is the representative of $f$ given by Theorem 3.6. Then, for any $\mathbf{x} \in \boldsymbol{\Omega}$ and any $0 \leqslant t<\tau_{-}(\mathbf{x})$

$$
f(\boldsymbol{\Phi}(\mathbf{x},-t))-f(\mathbf{x})=\int_{0}^{t}\left[\mathcal{T}_{\max } f\right](\mathbf{\Phi}(\mathbf{x},-r)) \mathrm{d} r
$$

which, according to the explicit expression of $U_{0}(t)$, means that

$$
U_{0}(t) f(\mathbf{x})-f(\mathbf{x})=\int_{0}^{t} U_{0}(r) \mathcal{T}_{\max } f(\mathbf{x}) \mathrm{d} r
$$

for any $\mathbf{x} \in \mathbf{\Omega}$ and $t<\tau_{-}(\mathbf{x})$. Letting $t$ converge towards $\tau_{-}(\mathbf{x})$ we obtain

$$
f(\mathbf{x})=-\int_{0}^{\tau_{-}(\mathbf{x})}\left[\mathcal{T}_{\max } f\right](\mathbf{\Phi}(\mathbf{x},-r)) \mathrm{d} r .
$$

In particular, Eq. (4.6) holds true for any $\mathrm{x} \in \boldsymbol{\Omega}$ and any $t \geqslant \tau_{-}(\mathbf{x})$. Arguing exactly as in [16, $\mathrm{p}$. 38], the pointwise identity (4.6) represents the $X$-integral, i.e, $U_{0}(t) f-f=\int_{0}^{t} U_{0}(r) \mathcal{T}_{\max } f \mathrm{~d} r$ in $L^{1}(\Omega, \mathrm{d} \mu)$. Consequently, $f \in \mathscr{D}\left(\mathcal{A}_{0}\right)$ with $\mathcal{A}_{0} f=\mathcal{T}_{\max } f$.

4.2. Green's formula. The above result allows us to treat more general boundary-value problem:

Theorem 4.2. Let $u \in L_{-}^{1}$ and $g \in X$ be given. Then the function

$$
f(\mathbf{x})=\int_{0}^{\tau_{-}(\mathbf{x})} \exp (-\lambda t) g(\boldsymbol{\Phi}(\mathbf{x},-t)) \mathrm{d} t+\chi_{\left\{\tau_{-}(\mathbf{x})<\infty\right\}} \exp \left(-\lambda \tau_{-}(\mathbf{x})\right) u\left(\mathbf{\Phi}\left(\mathbf{x},-\tau_{-}(\mathbf{x})\right)\right)
$$

is a unique solution $f \in \mathscr{D}\left(\mathcal{T}_{\max }\right)$ of the boundary value problem:

$$
\left\{\begin{array}{l}
\left(\lambda-\mathcal{T}_{\max }\right) f=g, \\
\mathrm{~B}^{-} f=u,
\end{array}\right.
$$


where $\lambda>0$. Moreover, $\mathrm{B}^{+} f \in L_{+}^{1}$ and

$$
\left\|\mathrm{B}^{+} f\right\|_{L_{+}^{1}}+\lambda\|f\|_{X} \leqslant\|u\|_{L_{-}^{1}}+\|g\|_{X},
$$

with equality sign if $g \geqslant 0$ and $u \geqslant 0$.

Proof. Let us write $f=f_{1}+f_{2}$ with $f_{1}(\mathbf{x})=\int_{0}^{\tau_{-}(\mathbf{x})} \exp (-\lambda t) g(\boldsymbol{\Phi}(\mathbf{x},-t)) \mathrm{d} t$, and

$$
f_{2}(\mathbf{x})=\chi_{\left\{\tau_{-}(\mathbf{x})<\infty\right\}} \exp \left(-\lambda \tau_{-}(\mathbf{x})\right) u\left(\mathbf{\Phi}\left(\mathbf{x},-\tau_{-}(\mathbf{x})\right)\right), \quad \mathbf{x} \in \mathbf{\Omega} .
$$

According to Theorem 4.1, $f_{1}=\left(\lambda-\mathcal{T}_{0}\right)^{-1} g$, i.e. $f_{1} \in \mathscr{D}\left(\mathcal{T}_{\max }\right)$ with $\left(\lambda-\mathcal{T}_{\max }\right) f_{1}=g$ and $\mathrm{B}^{-} f_{1}=0$. Therefore, to prove that $f$ is a solution of (4.7) it suffices to check that $f_{2} \in \mathscr{D}\left(\mathcal{T}_{\max }\right)$, $\left(\lambda-\mathcal{T}_{\max }\right) f_{2}=0$ and $\mathrm{B}^{-} f_{2}=u$. It is easy to see that $f_{2} \in L^{1}(\Omega, \mathrm{d} \mu)$ (see also (4.10)). To prove that $f_{2} \in \mathscr{D}\left(\mathcal{T}_{\text {max }}\right)$ one argues as in the proof of Theorem 4.1. Precisely, let $\psi \in \mathfrak{Y}$, noticing that $f_{2}$ vanishes outside $\Omega_{-}$, one has thanks to (4.9)

$$
\begin{aligned}
\left.\int_{\boldsymbol{\Omega}} f_{2}(\mathbf{x}) \frac{\mathrm{d}}{\mathrm{d} s} \psi(\mathbf{\Phi}(\mathbf{x}, s))\right|_{s=0} \mathrm{~d} \mu(\mathbf{x}) & =\int_{\Gamma_{-}} \mathrm{d} \mu_{-}(\mathbf{y}) \int_{0}^{\tau_{+}(\mathbf{y})} f_{2}(\mathbf{\Phi}(\mathbf{y}, t)) \frac{\mathrm{d}}{\mathrm{d} t} \psi(\boldsymbol{\Phi}(\mathbf{y}, t)) \mathrm{d} t \\
& =\int_{\Gamma_{-}} u(\mathbf{y}) \mathrm{d} \mu_{-}(\mathbf{y}) \int_{0}^{\tau_{+}(\mathbf{y})} \exp (-\lambda t) \frac{\mathrm{d}}{\mathrm{d} t} \psi(\mathbf{\Phi}(\mathbf{y}, t)) \mathrm{d} t .
\end{aligned}
$$

For almost every $\mathbf{y} \in \Gamma_{-}$, we compute the integral over $\left(0, \tau_{+}(\mathbf{y})\right)$ by parts, which yields $f_{2} \in$ $\mathscr{D}\left(\mathcal{T}_{\max }\right)$ with $\mathcal{T}_{\max } f_{2}=\lambda f_{2}$. Also,

$$
f_{2}(\mathbf{\Phi}(\mathbf{y}, t))=\exp (-\lambda t) u(\mathbf{y}), \quad \mathbf{y} \in \Gamma_{-}, \quad 0<t<\tau_{+}(\mathbf{y})
$$

from which we see that $\mathrm{B}^{-} f_{2}=u$.

Consequently, $f$ is a solution to (4.7). To prove that the solution is unique, it is sufficient to prove that the only solution $h \in \mathscr{D}\left(\mathcal{T}_{\max }\right)$ to $\left(\lambda-\mathcal{T}_{\max }\right) h=0, \mathrm{~B}^{-} h=0$, is $h=0$. This follows from the fact that such a solution $h$ actually belongs to $\mathscr{D}\left(\mathcal{T}_{0}\right)$ if $\lambda \in \varrho\left(\mathcal{T}_{0}\right)$. Finally, it remains to prove (4.8). For simplicity, we denote the representative of $f_{i}, i=1,2$, defined in Proposition 3.16, with the same letter. Using (4.9) and the fact that $f_{2}$ vanishes on $\boldsymbol{\Omega}_{-\infty}$, from (2.9) we get

$$
\begin{aligned}
\lambda \int_{\Omega}\left|f_{2}\right| \mathrm{d} \mu & =\lambda \int_{\Omega_{-}}\left|f_{2}\right| \mathrm{d} \mu=\lambda \int_{\Gamma_{-}} \mathrm{d} \mu_{-}(\mathbf{y}) \int_{0}^{\tau_{+}(\mathbf{y})} e^{-\lambda t}|u(\mathbf{y})| \mathrm{d} t \\
& =\int_{\Gamma_{-}}|u(\mathbf{y})|\left(1-e^{-\lambda \tau_{+}(\mathbf{y})}\right) \mathrm{d} \mu_{-}(\mathbf{y}) .
\end{aligned}
$$

Define $h: \mathbf{y} \in \Gamma_{-} \longmapsto h(\mathbf{y})=|u(\mathbf{y})| e^{-\lambda \tau_{+}(\mathbf{y})}$. It is clear that $h$ vanishes on $\Gamma_{-\infty}$ and $h(\mathbf{y}) \leqslant$ $|u(\mathbf{y})|$ for a.e. $\mathbf{y} \in \Gamma_{-}$. In particular, $h \in L_{-}^{1}$ and, according to (2.11),

$$
\begin{aligned}
& \int_{\Gamma_{-}} h(\mathbf{y}) \mathrm{d} \mu_{-}(\mathbf{y})=\int_{\Gamma_{-} \backslash \Gamma_{-}} h(\mathbf{y}) \mathrm{d} \mu_{-}(\mathbf{y})=\int_{\Gamma_{+} \backslash \Gamma_{+} \infty} h\left(\mathbf{\Phi}\left(z,-\tau_{-}(z)\right)\right) \mathrm{d} \mu_{+}(z) \\
& \quad=\int_{\Gamma_{+} \backslash \Gamma_{+} \infty} e^{-\lambda \tau_{-}(z)}\left|u\left(\mathbf{\Phi}\left(z,-\tau_{-}(z)\right)\right)\right| \mathrm{d} \mu_{+}(z)=\int_{\Gamma_{+}}\left|\mathrm{B}^{+} f_{2}(z)\right| \mathrm{d} \mu_{+}(z)=\left\|\mathrm{B}^{+} f_{2}\right\|_{L_{+}^{1}} .
\end{aligned}
$$

Combining this with (4.10) leads to

$$
\lambda\left\|f_{2}\right\|_{X}+\left\|\mathrm{B}^{+} f_{2}\right\|_{L_{+}^{1}}=\|u\|_{L_{-}^{1}} .
$$

Now, let us show that $\mathrm{B}^{+} f_{1} \in L_{+}^{1}$ and $\left\|\mathrm{B}^{+} f_{1}\right\|_{L_{+}^{1}}+\lambda\left\|f_{1}\right\|_{X} \leqslant\|g\|_{X}$. For any $\mathbf{y} \in \Gamma_{+}$and $0<t<\tau_{-}(\mathbf{y})$, we see, as above, that $f_{1}(\boldsymbol{\Phi}(\mathbf{y},-t))=\int_{t}^{\tau_{-}(\mathbf{y})} \exp (-\lambda(s-t)) g(\boldsymbol{\Phi}(\mathbf{y},-s)) \mathrm{d} s$. 
This shows that

$$
\left.\mathrm{B}^{+} f_{1}(\mathbf{y})=\lim _{t \rightarrow 0^{+}} f_{1}(\mathbf{\Phi}(\mathbf{y},-t))=\int_{0}^{\tau_{-}(\mathbf{y})} \exp (-\lambda s)\right) g(\mathbf{\Phi}(\mathbf{y},-s)) \mathrm{d} s .
$$

According to Proposition 2.12,

$$
\int_{\Gamma_{+}} \mathrm{d} \mu_{+}(\mathbf{y}) \int_{0}^{\tau_{-}(\mathbf{y})}|g(\mathbf{\Phi}(\mathbf{y},-s))| \mathrm{d} s=\int_{\boldsymbol{\Omega}_{+}}|g| \mathrm{d} \mu
$$

which, since $\exp (-\lambda(s-t))|g(\mathbf{\Phi}(\mathbf{y},-s))| \leqslant|g(\mathbf{\Phi}(\mathbf{y},-s))|$, implies $\mathrm{B}^{+} f_{1} \in L_{+}^{1}$. Let us now assume $g \geqslant 0$. Then $f_{1} \geqslant 0$ and hence

$$
\lambda\left\|f_{1}\right\|=\lambda \int_{\boldsymbol{\Omega}} f_{1} \mathrm{~d} \mu=\lambda \int_{\boldsymbol{\Omega}_{+}} f_{1} \mathrm{~d} \mu+\lambda \int_{\boldsymbol{\Omega}_{-} \cap \boldsymbol{\Omega}_{+\infty}} f_{1} \mathrm{~d} \mu+\lambda \int_{\boldsymbol{\Omega}_{-\infty} \cap \boldsymbol{\Omega}_{+}} f_{1} \mathrm{~d} \mu .
$$

Using similar arguments to those used in the study of $f_{2}$, we have

$$
\lambda \int_{\boldsymbol{\Omega}_{+}} f_{1} \mathrm{~d} \mu=\int_{\Gamma_{+}} \mathrm{d} \mu_{+}(\mathbf{y}) \int_{0}^{\tau_{-}(\mathbf{y})} g(\mathbf{\Phi}(\mathbf{y},-t))(1-\exp (-\lambda t)) \mathrm{d} t,
$$

which, by Proposition 2.12, implies $\lambda \int_{\Omega_{+}} f_{1} \mathrm{~d} \mu=\int_{\Omega_{+}} g \mathrm{~d} \mu-\int_{\Gamma_{+}} \mathrm{B}^{+} f_{1} \mathrm{~d} \mu_{+}$. Similar argument shows that $\lambda \int_{\boldsymbol{\Omega}_{-} \cap \boldsymbol{\Omega}_{+\infty}} f_{1} \mathrm{~d} \mu=\int_{\boldsymbol{\Omega}_{-} \cap \boldsymbol{\Omega}_{+\infty}} g \mathrm{~d} \mu$, while the equality

$$
\lambda \int_{\boldsymbol{\Omega}_{-\infty} \cap \boldsymbol{\Omega}_{+\infty}} f_{1} \mathrm{~d} \mu=\int_{\boldsymbol{\Omega}_{-\infty} \cap \boldsymbol{\Omega}_{+\infty}} g \mathrm{~d} \mu,
$$

is a direct consequence of the invariance of $\mu$ with respect to $\Phi(\cdot, t)$. This shows that $\lambda\|f\|_{X}=$ $\|g\|_{X}-\left\|\mathrm{B}^{+} f\right\|_{L_{+}^{1}}$ for $g \geqslant 0$. In general, defining

$$
F_{1}(\mathbf{x})=\int_{0}^{\tau_{-}(\mathbf{x})} \exp (-\lambda s) \mid g(\mathbf{\Phi}(\mathbf{x},-s) \mid \mathrm{d} s, \quad \mathbf{x} \in \mathbf{\Omega},
$$

we obtain $\left\|\mathrm{B}^{+} f_{1}\right\|_{L_{+}^{1}}+\lambda\left\|f_{1}\right\|_{X} \leqslant\left\|B^{+} F_{1}\right\|_{L_{+}^{1}}+\lambda\left\|F_{1}\right\|_{X}=\|g\|_{X}$ which, combined with (4.11), gives (4.8).

Remark 4.3. Notice that, in order to get the existence and uniqueness of the solution $f$ to (4.7), it is not necessary for $u$ to belong to $L^{1}\left(\Gamma_{-}, \mathrm{d} \mu_{-}\right)$. Indeed, we only have to make sure that $f_{2} \in L^{1}(\boldsymbol{\Omega}, \mathrm{d} \mu)$, i.e., from (4.10), $\int_{\Gamma_{-}}|u(\mathbf{y})|\left(1-e^{-\lambda \tau_{+}(\mathbf{y})}\right) \mathrm{d} \mu_{-}(\mathbf{y})<\infty$. Of course, to get (4.8), the assumption $u \in L^{1}\left(\Gamma_{-}, \mathrm{d} \mu_{-}\right)$is necessary.

Let us note that, with the notation of Theorem 4.2, we have

$$
\int_{\Gamma_{+}} \mathrm{B}^{+} f \mathrm{~d} \mu_{+}+\lambda \int_{\Omega} f \mathrm{~d} \mu=\int_{\Gamma_{-}} u \mathrm{~d} \mu_{-}+\int_{\Omega} g \mathrm{~d} \mu .
$$

Indeed, for nonnegative $u$ and $g$, (4.8) turns out to be precisely (4.12). Then, for arbitrary $u \in L_{-}^{1}$ and $g \in X$, we get (4.12) by splitting functions into positive and negative parts. This leads to the following generalization of Green's formula:

Proposition 4.4 (Green's formula). Let $f \in \mathscr{D}\left(\mathcal{T}_{\max }\right)$ satisfies $\mathrm{B}^{-} f \in L_{-}^{1}$. Then $\mathrm{B}^{+} f \in L_{+}^{1}$ and

$$
\int_{\Omega} \mathcal{T}_{\max } f \mathrm{~d} \mu=\int_{\Gamma_{-}} \mathrm{B}^{-} f \mathrm{~d} \mu_{-}-\int_{\Gamma_{+}} \mathrm{B}^{+} f \mathrm{~d} \mu_{+}
$$


Proof. For given $f \in \mathscr{D}\left(\mathcal{T}_{\max }\right)$, we obtain the result by setting $u=\mathrm{B}^{-} f \in L_{-}^{1}$ and $g=(\lambda-$ $\left.\mathcal{T}_{\max }\right) f \in X$ in Eq. (4.12).

Remark 4.5. If $\mathrm{d} \mu$ is the Lebesgue measure on $\mathbb{R}^{N}$, the above formula leads to a better understanding of the measures $\mathrm{d} \mu_{ \pm}$. Indeed, comparing it to the classical Green's formula (see e.g. [8]), we see that the restriction of $\mathrm{d} \mu_{ \pm}$on the set $\Sigma_{ \pm}=\{\mathbf{y} \in \partial \boldsymbol{\Omega} ; \pm \mathscr{F}(\mathbf{y}) \cdot n(\mathbf{y})>0\}$ equals

$$
|\mathscr{F}(\mathbf{y}) \cdot n(\mathbf{y})| \mathrm{d} \gamma(\mathbf{y})
$$

where $\mathrm{d} \gamma(\cdot)$ is the surface Lebesgue measure on $\partial \boldsymbol{\Omega}$.

\section{APPENDiX: About the Class OF TEST-FUnCTIONS}

We answer in this Appendix a natural question concerning the definition of the class of testfunctions $\mathfrak{Y}$. Precisely, we prove that two test-functions equal $\mu$-almost everywhere are such that their derivatives (in the sense of (3.1)) also coincide $\mu$-almost everywhere. To prove our claim, it clearly suffices to prove that, given $\psi \in \mathfrak{Y}$ such that $\psi(\mathbf{x})=0$ for $\mu$-a. e. $\mathbf{x} \in \boldsymbol{\Omega}$, one has $\varphi(\mathbf{x})=0$ for $\mu$-a. e. $\mathbf{x} \in \boldsymbol{\Omega}$ where $\varphi(\mathbf{x})=\left.\frac{\mathrm{d}}{\mathrm{d} s} \psi(\mathbf{\Phi}(\mathbf{x}, s))\right|_{s=0}$. Let

$$
E:=\{\mathbf{x} \in \boldsymbol{\Omega} ; \psi(\mathbf{x})=0 \text { and } \varphi(\mathbf{x}) \neq 0\} .
$$

It is clear that $E$ is measurable and that one has to prove that $\mu(E)=0$. It is no loss of generality to assume that $E$ is bounded. We observe that for any $\mathbf{x} \in E$, there exists $\delta_{\mathbf{x}}>0$ such that

$$
\psi(\mathbf{\Phi}(\mathbf{x}, t)) \neq 0, \quad \forall 0<|t|<\delta_{\mathbf{x}} .
$$

Let us split $E$ as follows

$$
E=\left(E \cap \boldsymbol{\Omega}_{-}\right) \cup\left(E \cap \boldsymbol{\Omega}_{+} \cap \boldsymbol{\Omega}_{-\infty}\right) \cup\left(E \cap \boldsymbol{\Omega}_{+\infty} \cap \boldsymbol{\Omega}_{-\infty}\right):=E_{-} \cup E_{+} \cup E_{\infty}
$$

and prove that $\mu\left(E_{-}\right)=\mu\left(E_{+}\right)=\mu\left(E_{\infty}\right)=0$.

(1) First consider $E_{-}$. Since $\psi(\mathbf{x})=0$ for $\mu$-a. e. $\mathbf{x} \in \boldsymbol{\Omega}_{-}$and using the fact that any $\mathbf{x} \in \boldsymbol{\Omega}_{-}$ can be written as $\mathbf{x}=\boldsymbol{\Phi}(\mathbf{y}, t)$ for some $\mathbf{y} \in \Gamma_{-}$and $0<t<\tau_{+}(\mathbf{y})$, we observe that, for $\mu_{-}$a. e. $\mathbf{y} \in \Gamma_{-}, \psi(\boldsymbol{\Phi}(\mathbf{y}, t))=0$ for almost every (in the sense of the Lebesgue measure in $\mathbb{R}) 0<t<\tau_{+}(\mathbf{y})$. For such a $\mathbf{y} \in \Gamma_{-}$, continuous differentiability of $t \mapsto \psi(\mathbf{\Phi}(\mathbf{y}, t))$ implies $\psi(\mathbf{\Phi}(\mathbf{y}, t))=0$ for any $0<t<\tau_{+}(\mathbf{y})$. This means, according to (A.1) that, for $\mu_{-}$-a. e. $\mathbf{y} \in \Gamma_{-}, \boldsymbol{\Phi}(\mathbf{y}, t) \notin E$ for any $0<t<\tau_{+}(\mathbf{y})$. Since

$$
\mu\left(E \cap \boldsymbol{\Omega}_{-}\right)=\int_{\Gamma_{-}} \mathrm{d} \mu_{-}(\mathbf{y}) \int_{0}^{\tau_{+}(\mathbf{y})} \chi_{E}(\mathbf{\Phi}(\mathbf{y}, t)) \mathrm{d} t
$$

we see that $\mu\left(E_{-}\right)=0$.

(2) In the same way, using $\Gamma_{+}$instead of $\Gamma_{-}$, we show that $\mu\left(E \cap \boldsymbol{\Omega}_{+} \cap \boldsymbol{\Omega}_{-\infty}\right)=0$.

(3) It remains to prove that $\mu\left(E_{\infty}\right)=0$. In accordance with (A.1), we define for, any $n \in \mathbb{N}$,

$$
E_{n}:=\left\{\mathbf{x} \in E_{\infty} ; \delta_{\mathbf{x}} \geqslant 1 / n\right\}=\left\{\mathbf{x} \in E_{\infty} ; \psi(\mathbf{\Phi}(\mathbf{x}, t)) \neq 0, \forall 0<|t|<1 / n\right\} .
$$

According to Assumption 1 , it is easy to see that $\mu\left(E_{n}\right)=0$ for any $n \in \mathbb{N}$ since $\psi(\mathbf{x})=0$ for $\mu$-a.e. $\mathbf{x} \in \Omega$. Moreover, $E_{1} \subset E_{2} \subset \ldots \subset E_{n} \subset E_{n+1} \subset \ldots$, and

$$
\bigcap_{n=1}^{\infty}\left(E_{\infty} \backslash E_{n}\right)=\varnothing \text {. }
$$


Since we assumed $\mu(E)<\infty$, we have $\mu\left(E_{\infty} \backslash E_{1}\right)<\infty$ and $\lim _{n \rightarrow \infty} \mu\left(E_{\infty} \backslash E_{n}\right)=0$. Writing $E_{\infty}=E_{n} \cup\left(E_{\infty} \backslash E_{n}\right)$, we see that $\mu\left(E_{\infty}\right)=0$.

\title{
REFERENCES
}

[1] H. Amann, Ordinary Differential Equations. An introduction to nonlinear analysis, W. de Gruyter, Berlin, 1990.

[2] L. Ambrosio, Transport equation and Cauchy problem for $B V$ vector fields, Invent. Math. 158 227-260, 2004.

[3] L. Ambrosio, Transport equation and Cauchy problem for non-smooth vector fields, Lecture Notes in Mathematics "Calculus of Variations and Non-Linear Partial Differential Equations" (CIME Series, Cetraro, 2005) 1927, B. Dacorogna, P. Marcellini eds., 2-41, 2008.

[4] L. Arlotti, B. Lods, Substochastic semigroups for transport equations with conservative boundary conditions, J. Evol. Equations 5 485-508, 2005.

[5] L. Arlotti, J. Banasiak, B. Lods, On transport equations driven by a non-divergence-free force field, Math. Meth. Appl. Sci., 30 2155-2177, 2007

[6] _ On general transport equations with abstract boundary conditions. The case of divergence free force field, preprint, 2008.

[7] J. Banasiak, L. Arlotti, Perturbations of positive semigroups with applications, Springer-Verlag, 2006.

[8] C. Bardos, Problèmes aux limites pour les équations aux dérivées partielles du premier ordre à coefficients réels; théorèmes d'approximation; application à l'équation de transport. Ann. Sci. École Norm. Sup. 3 185233, 1970.

[9] R. Beals, V. Protopopescu, Abstract time-dependent transport equations, J. Math. Anal. Appl. 121 370-405, 1987.

[10] R. DiPerna, P.-L. Lions, Ordinary differential equations, transport theory and Sobolev spaces. Invent. Math. 98 511-547, 1989.

[11] W. Greenberg, C. van der Mee and V. Protopopescu, Boundary Value Problems in Abstract Kinetic theory, Birkhäuser Verlag, Basel, 1987.

[12] P. R. Halmos, Measure Theory, Van Nostrand, Toronto, 3rd ed., 1954.

[13] C. Le Bris, P.-L. Lions, Renormalized solutions of some transport equations with partially $W^{1,1}$ velocities and applications, Ann. Mat. Pura Appl. 183 97-130, 2004.

[14] C. van der Mee, Time dependent kinetic equations with collision terms relatively bounded with respect to collision frequency, Transp. Theory Stat. Phys. 30 63-90, 2001.

[15] H. L. Royden, Real Analysis, Macmillan, New York, 3rd ed., 1988.

[16] J. Voigt, Functional analytic treatment of the initial boundary value problem for collisionless gases, München, Habilitationsschrift, 1981.

\author{
Luisa Arlotti \\ Dipartimento di Ingegneria Civile, Università di Udine, Via Delle Scienze 208, \\ 33100 UDINE, ITALY. \\ luisa.arlotti@uniud.it
}

\section{Jacek Banasiak}

School of Mathematical Sciences, University of KwaZulu-Natal,

DURban 4041, South AFricA.

banasiak@ukzn.ac.za

\section{Bertrand Lods}

Laboratoire de Mathématiques, CNRS UMR 6620, Université Blaise Pascal (Clermont-FerRand 2), 63177 Aubière Cedex, France.

bertrand. lods@math. univ-bpclermont. fr 